\title{
Psychosomatische Störungen im Kindes- und Jugendalter
}

\author{
Stephan Anis Towfigh*, Lena Kuntze*, Michael von Aster
}

\section{Übersicht}

\section{Einleitung}

Entwicklungspsychologische und neurobiologische Grundlagen

zum Verständnis

psychosomatischer Störungen

Definition, Klassifikation und

klinisches Erscheinungsbild

\section{1}

112

117

\section{Einleitung}

„Schon wieder dieser Junge, dem kann ich doch nicht helfen“, „Oje, das passt heute ja überhaupt nicht rein“, „Der geb' ich schnell 'ne Krankschreibung, hoffentlich löchert sie mich nicht mit Fragen“ oder „Warum gibt diese Mutter denn keine Ruhe mit dem Kind?“-Vielleicht fühlen Sie sich beim Lesen solcher Gedanken an Gefühle von Verunsicherung, Überforderung, Ärger oder gar Wut erinnert, wenn Ihnen Patienten mit sogenannten Psychosomatischen Störungen in den Sinn kommen.

Schätzungen zur Folge haben somatoforme Störungen eine Lebenszeitprävalenz von 13,1\% [1], für funktionelle Störungen liegt die Lebenszeitprävalenz sogar bei $25 \%$ [2]. Bei der Betrachtung von Patienten mit langwierigen chronischen Verläufen somatoformer Störungen im Erwachsenenalter geben 75\% einen Beginn ihrer Beschwerden vor dem 20. Lebensjahr an [3, 4]. Vor diesem Hintergrund kommt Kinderärzten eine besondere Bedeutung bei der Ersteinschätzung des Störungsbildes, bei differenzialdiagnostischen Erwägungen und bei der weichenstellenden Einleitung therapeutischer Maßnahmen zu.

Der enge und vielschichtige Zusammenhang zwischen körperlichem und psychischem Befinden ist offensichtlich und für Jedermann alltäglich spürbar. Und

* Die Autoren trugen in gleichem Maße zur Veröffentlichung bei. obwohl dies nicht nur für Zustände von Wohlbefinden („....ich könnte Bäume ausreißen!“), sondern auch für Zustände von Unbehagen gilt ( „....mir wird übel und ich bekomme Bauchkrämpfe, wenn ich nur dran denke...“ als Ausdruck von Angst), ist ein körperliches Symptom oft leichter zu akzeptieren als ein psychisches. Hier spielen Wahrnehmungs- und Bewertungsprozesse eine entscheidende Rolle, und zwar sowohl die eigenen als auch jene der umgebenden Menschen.

Schon erste Erfahrungen beim Durchmachen und Bewältigen einfacher Kinderkrankheiten (z. B. Infekte) können dem Betroffenen das Phänomen des sogenannten Krankheitsgewinns und die Möglichkeiten zu seiner Instrumentalisierung erfahrbar machen. Dies kann der Fall sein, wenn beispielsweise die Angst vor einer Klassenarbeit am nächsten Schultag zeitlich mit einem Magen-Darm-Infekt zusammenfällt und so das erkrankungsbedingte Zuhausebleiben-Müssen auch den Aspekt eines Zuhausebleiben-Dürfens erhältdurch die damit verbundene Reduktion der Angst vor der Klassenarbeit. Dieser primäre Krankheitsgewinn kann noch durch einen sekundären verstärkt werden, indem das Kind nun zuhause besondere Zuwendung, Pflege oder stimulierende Ablenkung erfährt. Die Versuchung, Krankheit in diesem Sinne zu nutzen, steht in einem engen Zusammenhang mit Haltungen und Einstellungen des Kindes, für deren Ausrichtung aber elterliche Modelle und Bewertungen ausschlaggebend sind. Davon hängt ab, ob die auf die krankheitsbedingt 
vermiedene Klassenarbeit bezogene Erleichterung als unerlaubt empfunden und vielleicht verschwiegen werden muss, oder ob das schulische Problem und das eigene Krankheitserleben in einer unterstützenden und feinfühligen Elternbeziehung kommuniziert und abgewogen werden kann. Wird die Ambivalenz des Kindes nicht wahrgenommen und die Krankheitsposition einseitig bekräftigt, so kann dies die Entstehung einer Somatisierungsstörung begünstigen.

In der Allgemein- oder Kinderärztlichen Praxis sind solche Patientenkontakte oft kräftezehrend, benötigen Geduld und Gespräch - und damit eine Ressource, die angesichts der heutigen Kostenstrukturen eng bemessen ist: Zeit.

In der vorliegenden Übersicht haben wir zu Beginn einen besonderen Akzent auf die Darstellung entwicklungspsychologischer und neurobiologischer Grundlagen für das Verständnis psychosomatischer Störungen gelegt. Im darauf folgenden Abschnitt gehen wir auf die Definition, die Klassifikation und die klinischen Erscheinungsformen der Dissoziativen Störungen und der Somatoformen Störungen ein, um schließlich einige Schlussfolgerungen und Anleitungen für die Praxis zu geben und mit kurzen Fallskizzen zu veranschaulichen.

\section{Entwicklungspsychologische und neurobiologische Grund- lagen zum Verständnis psycho- somatischer Störungen}

Die gesunde Entwicklung einer psychischen und kör-

Abb. 1 Ein Säugling weint, weil er Hunger hat. Je älter das Kind, desto mehr rückt die „sprachliche Antwort" vor die „handelnde Antwort", bis Kinder schließlich in der Lage sind, das nagende Gefühl im oberen Bauch einzuordnen und selbst sagen zu können, dass sie Hunger haben. Symbolbild, Quelle: Thieme Verlagsgruppe, Galina Barskaya. perlichen Integration erfordert eine Reihe von wesentlichen Erfahrungen in der kindlichen Entwicklung. Sie bilden die Grundlage für das psychische Gleichgewicht und spiegeln notwendige Werkzeuge für die Ent-

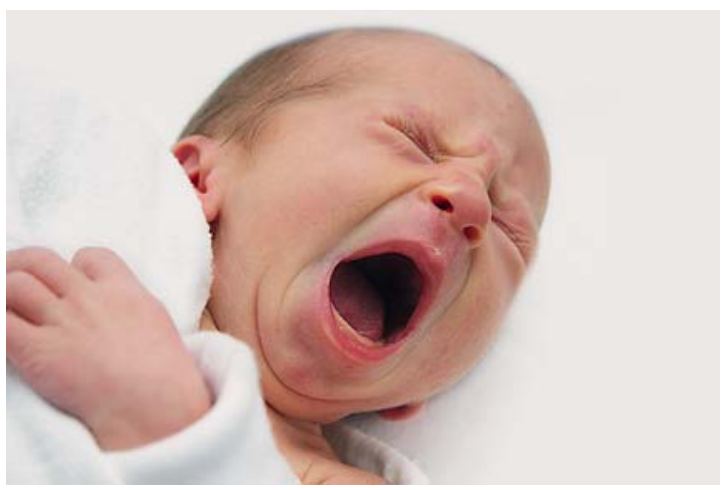

schlüsselung der Erlebniswelt wider. Gleichzeitig sind es Erfahrungen, die das gesamte Leben hindurch gestärkt und erweitert werden.

Einen guten Überblick über diese zentralen Erfahrungen gibt Rudolf [5] wie folgt:

- Erfahrung, sich ausdrücken zu können und das wichtige Gegenüber damit auch zu erreichen,

- Erfahrung, als Antwort auf den eigenen Appell, beachtet, verstanden, beruhigt, getröstet zu werden (wichtige Grundlage der späteren Selbstberuhigung),

- Erfahrung, Situationen gemeinsam emotional bewerten und tragen zu können (Entstehung des „Wir-Gefühls“),

- sprachlich begriffliche Differenzierung von Emotionen,

- Erfahrung des Körper-Selbst und der Differenzierung (gemeinsame Bewertung von Körpererfahrungen, wie innerer und äußerer Schmerz),

- Erfahrung „Das bin ich, das bist Du“: Differenzierung zwischen Ich und Nicht-Ich.

Zum Verständnis der Schmerzwahrnehmung und -interpretation von Patienten mit somatoformen Störungen ist zunächst die Entwicklung der Körperwahrnehmungen im Allgemeinen zu betrachten. Einerseits geht es dabei um die Wahrnehmung von körperlichen Zuständen wie Hunger und Kälte, andererseits um die Wahrnehmung von Emotionen und der damit verbundenen Körperreaktionen.

Merke: Während der ersten Lebensmonate und -jahre benötigen Säuglinge und Kleinkinder die Hilfe ihrer Umgebung, um die Welt, in der sie leben, zu verstehen.

Dabei sind sie darauf angewiesen, dass ihre Empfindungen und Bedürfnisse durch nahe Bezugspersonen (zumeist die Eltern) gespiegelt und sprachlich markiert (also „verbalisiert“) werden. Ebenso wie Sprache nur durch Ansprache entstehen kann, sind Kinder für die Einordnung von körperlichen Zuständen und von Gefühlen zunächst also auf die Einordnung und Benennung durch die Eltern angewiesen.

\section{Entwicklung des Körpergefühls}

Säuglinge weinen beispielsweise, weil sie Hunger haben. In einer positiven, gelingenden Eltern-KindInteraktion wird die Mutter hierauf nicht nur reagieren, indem sie den Säugling stillt, sondern auch indem sie ihm mitteilt: „Du hast Hunger.“ Je älter die Kinder 
werden, desto mehr rückt die „sprachliche Antwort“ vor die "handelnde Antwort“, bis Kinder schließlich in der Lage sind, das nagende Gefühl im oberen Bauch einzuordnen und selbst sagen zu können: „Mama, ich habe Hunger“ (Abb.1).

Diesem Prinzip folgend gelingt es einem Kind zunehmend, körperlichen Zuständen innere Bilder und Empfindungen zuzuordnen und Sicherheit im Umgang mit dem eigenen Körper zu gewinnen. Auch Einflüsse von außen, die auf den Körper einwirken, wie Kälte und Wärme, können so erkannt und eingeordnet werden. Der nicht nur mechanisch versorgende, sondern auch spielerisch liebkosende Körperkontakt belebt die Körpererfahrung von Säuglingen und Kleinkindern und fördert eine Verknüpfung von Körpererleben (Wohlgefühl) und Emotion (Glück). Gelingt diese Entwicklung, entsteht eine lebendige, gesunde Wahrnehmung, das sogenannte „Körper-Selbst“ (Abb.2).

Merke: Sind Eltern oder die erziehenden Bezugspersonen durch starke Überforderung oder eigene psychische und körperliche Beeinträchtigungen nicht in der Lage, körperliche Zustände und Bedürfnisse beim Kind wahrzunehmen und angemessen darauf zu reagieren, kann sich eine gestörte Wahrnehmung des eigenen Körpers entwickeln.

In Bezug auf somatoforme Störungen haben hier häufig Fehlwahrnehmungen oder Fehlinterpretationen, wie die Einordnung von Völlegefühl als Bauchschmerz, ihren Ursprung. Auch bei anderen psychiatrischen Erkrankungen, z.B. bei Essstörungen, kann das Körperselbst deutlich beeinträchtigt sein.

Eine weitere wesentliche Lernaufgabe für ein Kleinkind ist, dass manche körperliche Zustände und Bedürfnisse wie Hunger und Müdigkeit zyklischen Rhythmen folgen, die sich der eigenen Kontrolle entziehen und auf die es zu reagieren gilt. Patienten mit somatoformen Störungen haben häufig das Gefühl, die Kontrolle zu verlieren, wenn sie sich diesen Rhythmen hingeben und missachten sie häufig.

\section{Entwicklung der Gefühlswelt}

Zur Betrachtung der Gefühlswelt im Kontext der kindlichen Entwicklung lassen sich Gefühle in zwei Teilaspekte untergliedern: zum einen die Wahrnehmung des eigentlichen Gefühls oder der Stimmung, und zum anderen die Wahrnehmung der damit einhergehenden und sie begleitenden körperlichen Reaktionen, wie

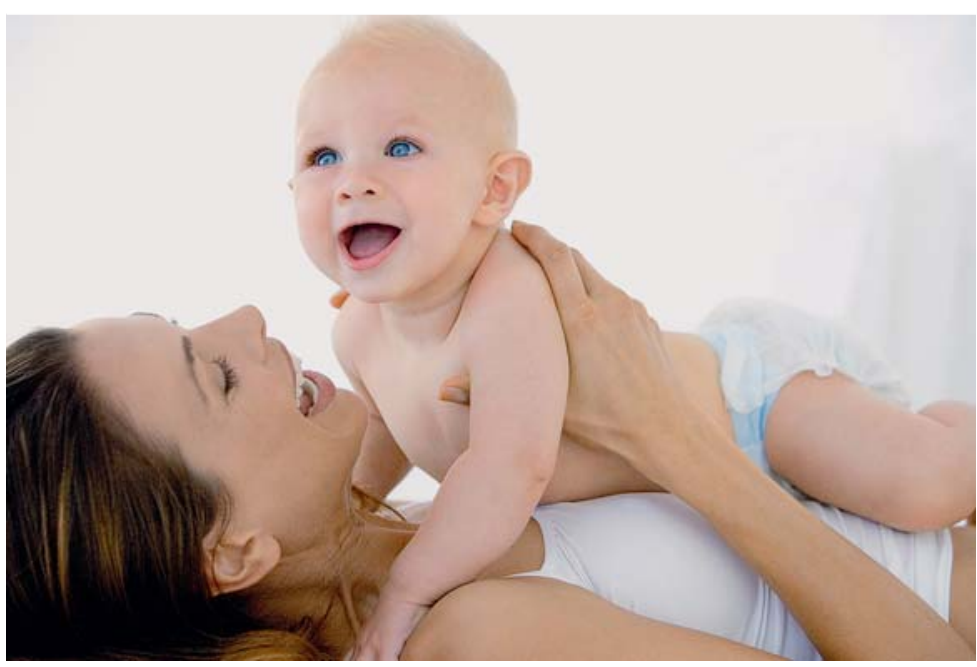

Abb. 2 Der nicht nur mechanisch versorgende, sondern auch spielerisch liebkosende Körperkontakt belebt die Körpererfahrung von Säuglingen und Kleinkindern und fördert eine Verknüpfung von Körpererleben und Emotion. Symbolbild, Quelle: Thieme Verlagsgruppe.

Herzklopfen bei Angst. Ähnlich wie bei der Wahrnehmung körperlicher Zustände sind Säuglinge und Kinder auf ihre Bezugspersonen angewiesen, um eine Differenzierung ihrer Gefühlszustände zu erlernen.

Merke: Für eine stabile und gesunde Entwicklung benötigen Säuglinge ein haltgebendes und kontinuierliches Gegenüber.

Sie können sich zunächst über Schreien, Weinen, Suchen von Blickkontakt, Abwenden etc. mitteilen und bedürfen eines Erwachsenen, der ihre Bedürfnisse und Zustände wahrnimmt, sie richtig interpretiert und eine prompte und angemessene Antwort geben kann („Feinfühligkeit“ nach Ainsworth [6]). Je mehr Mitteilungsmöglichkeiten ein Säugling nutzt, desto leichter wird es auch für die Bezugspersonen sein, zu reagieren. Diese Antwort kann aus einer Handlung oder einer verbalen Antwort bestehen, im Idealfall aus beidemäquivalent der Reaktion auf körperliche Grundbedürfnisse.

Erschreckt sich beispielsweise ein Säugling, weil ein lautes Geräusch zu hören war, so muss die Bezugsperson zunächst wahrnehmen, dass das Kind erschreckt wurde. Anschließend muss ein rascher innerlicher Abgleich darüber folgen, wie die Situation eingeordnet wird: Droht eine reale Gefahr, oder handelt es sich um eine harmlose Störung des Ablaufs? Als passende und prompte Antwort folgen dann in einer ungefährlichen Situation die körperliche Beruhigung des Kindes (Schaukeln etc.) und eine verbale Begleitung, wie „Das war laut, da hast du dich erschreckt“ (Abb.3). 
Abb.3 Gefühle wahrnehmen und adäquat antworten. Symbolbild, Quelle: Thieme Verlagsgruppe.

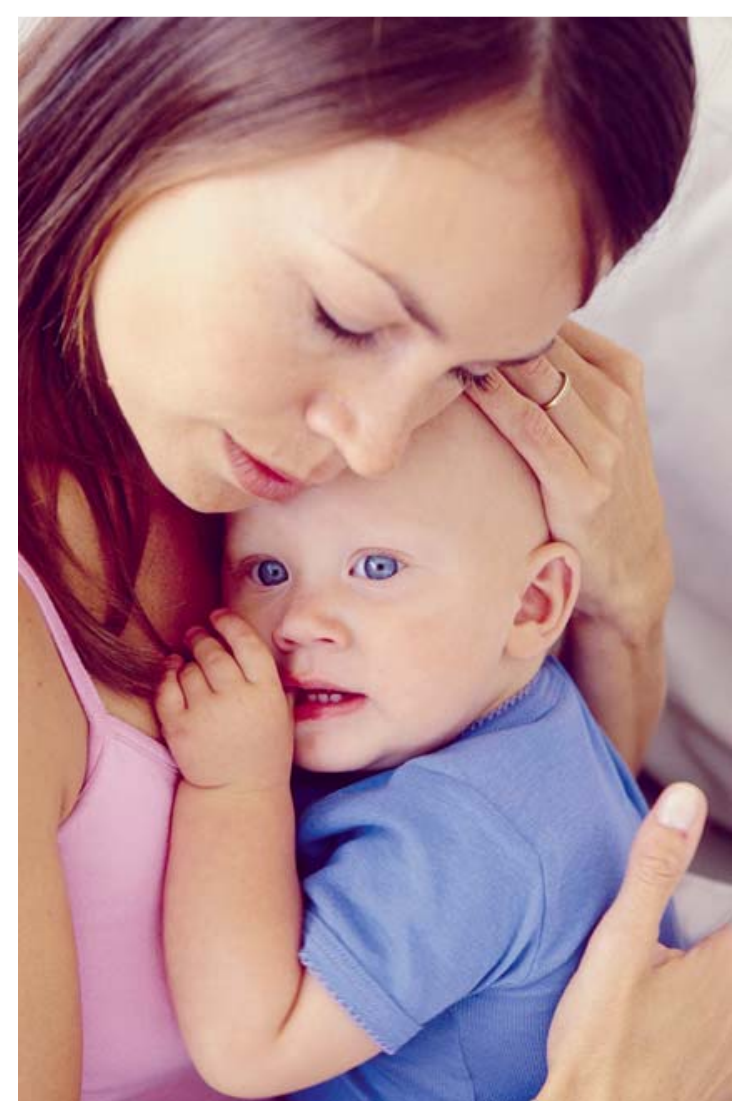

Bezugspersonen müssen also in der Lage sein, eine innere Einordnung der Situation vorzunehmen, diese abzugleichen und eine angemessene Antwort zu finden. Globale Antworten wie: „Ist nicht schlimm, ist ja nichts passiert.“, greifen häufig zu kurz, ermöglichen dem Kind keinen Abgleich mit seiner inneren Verfassung und führen zu keiner Beruhigung. Die Reaktionen der Betreuungspersonen haben also einen unmittelbaren Einfluss darauf, wie das Kind ein Gefühl erlebt und verarbeitet.

\section{Störungen im Entwicklungsablauf}

Merke: Je vorhersehbarer die Reaktionen der Bezugspersonen sind, desto eher gelingt es den Säuglingen und Kleinkindern ein Bewusstsein für ihre eigenen inneren Zustände zu entwickeln.

In manchen Fällen können sich primäre Bindungspersonen nicht gut genug vom kindlichen Affekt abgrenzen. Dies kann beispielsweise bei psychischer oder körperlicher Erkrankung oder Traumatisierung der Eltern der Fall sein, aber auch bei erheblicher psychosozialer Belastung auftreten. Anstelle einer angemessen Bewertung einer Gefühlsregung des Kindes wird die Bindungsperson dann von der Verzweiflung des Kindes angesteckt und kann daraufhin nicht mehr adäquat beruhigen oder trösten.

Das emotionale Erleben und Handeln der Bezugspersonen spiegelt sich im emotionalen Erleben und Handeln des Kindes wider. Der elterliche Affekt ist für das Verständnis des Kindes entscheidend, er signalisiert entweder Entwarnung und Beruhigung oder die Existenz von Gefahr, auf die das Kind mit Schreck, Angst und Weinen reagieren wird.

Eltern können beispielsweise durch wahnhafte, aber auch neurotische Zustände Fehleinschätzungen von Gefahren vornehmen und anstelle von Beruhigung eher für eine Eskalation sorgen. Ebenso können sie sich in einer dauerhaften, nicht veränderbaren (z. B. ängstlichdepressiven) Stimmung befinden, die ihre Wahrnehmung und damit auch ihre Antworten einschränkt und dazu führt, dass kindliche Signale gar nicht erst wahrgenommen werden.

Bei Suchterkrankungen, psychotischen Erkrankungen oder Persönlichkeitsstörungen der Bezugspersonen kann die Reaktion sehr wechselhaft und unvorhersehbar für ein Kind sein: Sie schwankt zuweilen zwischen angemessener Reaktion, Widersprüchlichkeit und gänzlichem Ausbleiben einer Reaktion. Dies führt nachvollziehbarerweise zu einer hohen Verunsicherung eines Kindes.

Sind Bezugspersonen durch eigene Beeinträchtigungen also nicht in der Lage, die Situation realistisch einzuordnen und angemessen zu reagieren, wird sich beim Kind ein unzureichendes Vertrauen in die Wahrnehmung eigener Zustände und in die Äußerung eigener Bedürfnisse entwickeln. Fehl- und Mangelentwicklung können also auch als eine mangelhafte Passung zwischen den Versorgungsmöglichkeiten der Bindungspersonen und den Grundbedürfnissen des Kindes verstanden werden.

\section{Verinnerlichung biografischer Erfahrungsmuster}

Merke: Die Erfahrung von gelingender Regulierung innerer Zustände in der Interaktion zwischen Bindungspersonen und Kind ist eine wichtige Voraussetzung für die spätere Fähigkeit zur Selbstregulierung. 
Psychologische Fehladaptation bei Kindern und Eltern (vgl. [7])

$\begin{array}{lcc}\begin{array}{l}\text { 1. Katastrophisierende Schmerzverarbei- } \\ \text { tung (bei Kind und/oder Eltern) }\end{array} & \begin{array}{c}\text { 2. Dysfunktionale Eltern-Kind-Arzt- } \\ \text { Kommunikation }\end{array} & \begin{array}{c}\text { Anzweifeln des Schmerzes auf Seiten } \\ \text { der Eltern und folgende Aggravie- }\end{array} \\ \text { - Übersteigerung der Symptomatik } & \text { 3. Dysfunktionale Schmerzkommunika- } & \begin{array}{l}\text { rung beim Kind, um nicht das Gesicht } \\ \text { - Generalisierung der Symptomatik }\end{array} \\ \begin{array}{l}\text { - subjektive Hilflosigkeit gegenüber } \\ \text { Beschwerden }\end{array} & \begin{array}{c}\text { tion zwischen Eltern und Kind } \\ \text { - Aggravierung und übermäßige Inan- }\end{array} & \\ \text { - Aufmerksamkeitsfokussierung } & \text { spruchnahme medizinischer Hilfen } & \end{array}$

Hier sind sowohl die Gefühlswahrnehmung („Ich bin nicht nur traurig, sondern auch wütend“), als auch die Entschlüsselung von Körpersignalen („Mein Herz klopft, weil ich aufgeregt bin“) Voraussetzung für die eigene Regulationsfähigkeit. Dabei lernt ein Kind nicht nur, körperliche Bedürfnisse mitzuteilen. Es lernt zunehmend, einen Austausch über das Gefühlsleben vorzunehmen, der wiederum das Empfinden von Zugehörigkeit vermittelt, das „Wir-Gefühl“.

Patienten mit somatoformen Störungen fehlt häufig die Erfahrung, sich so ausdrücken zu können, dass andere damit emotional angemessen erreicht werden. Eine mangelnde Antwort von Bezugspersonen kann zu einem Mangel an dem Gefühl führen, dass andere es gut mit einem meinen. Zudem verzögert sich die Entwicklung von emotionalem Verständnis, was zu einer spezifischen Form emotionaler Spracharmut und Sprachlosigkeit führt. Äußerungen wie: „Ich fühle mich unter Druck“, ohne dass ein Zusammenhang zu aktuellen belastenden Erlebnissen hergestellt werden kann, sind typisch für Menschen mit somatoformen Zustandsbildern.

Andererseits begünstigen unangemessene oder überbesorgte Reaktionen der Eltern, z. B. übertriebene Schonung oder Vereinbarung häufiger Arzttermine und Untersuchungen, einen psychosomatischen Krankheitsverlauf. Das Kind erlebt die Sorge und die Zuwendung als Beziehungsgewinn („sekundärer Krankheitsgewinn“) und verinnerlicht ein Konzept ernsthafter körperlicher Erkrankung (siehe Kasten: Psychologische Fehladaption).

\section{Reifungsanforderungen im Jugendalter}

Mit den frühen Erfahrungen und den im Kindesalter entwickelten Fähigkeiten muss das Kind nun die Entwicklungsaufgaben des Jugendalters bewältigen. In zunehmendem Maße stehen ihm reifere kognitive Fertigkeiten zur Verfügung, die es dazu befähigen, Erfahrungen, situative Kontexte, eigene Gefühle und dieje- nigen anderer Personen bewusster wahrzunehmen, zu benennen und zu reflektieren.

Der Prozess der Selbstständigkeit des Kindes nimmt im Jugendalter zunehmend die Qualität der Verantwortungsübernahme für die Alltagsbewältigung einerseits und für die Gefühlsverarbeitung andererseits an. Die Frontalhirnentwicklung, die in starkem Zusammenhang mit der Entwicklung der Aufmerksamkeitslenkung, Handlungsplanung, Gefühlsregulierung und Steuerungsfähigkeit steht, ist im Jugendalter so ausgeprägt wie nie vorher.

Im Zusammenspiel mit der körperlichen Reifung und der Auseinandersetzung mit dem inneren und äußeren Selbstbild nehmen auch charakterliche Ausprägungen zunehmend Gestalt an und können im Rahmen der gesteigerten kognitiven Reflexionsfähigkeit besser überprüft und weiterentwickelt werden. Fragen der Positionierung in der Gleichaltrigengruppe, die Ausgestaltung eines eigenen Wertesystems und schließlich die Entwicklung von gesundem Zutrauen in die eigenen Fähigkeiten sind Aufgaben der Adoleszenzentwicklung (Abb.4).

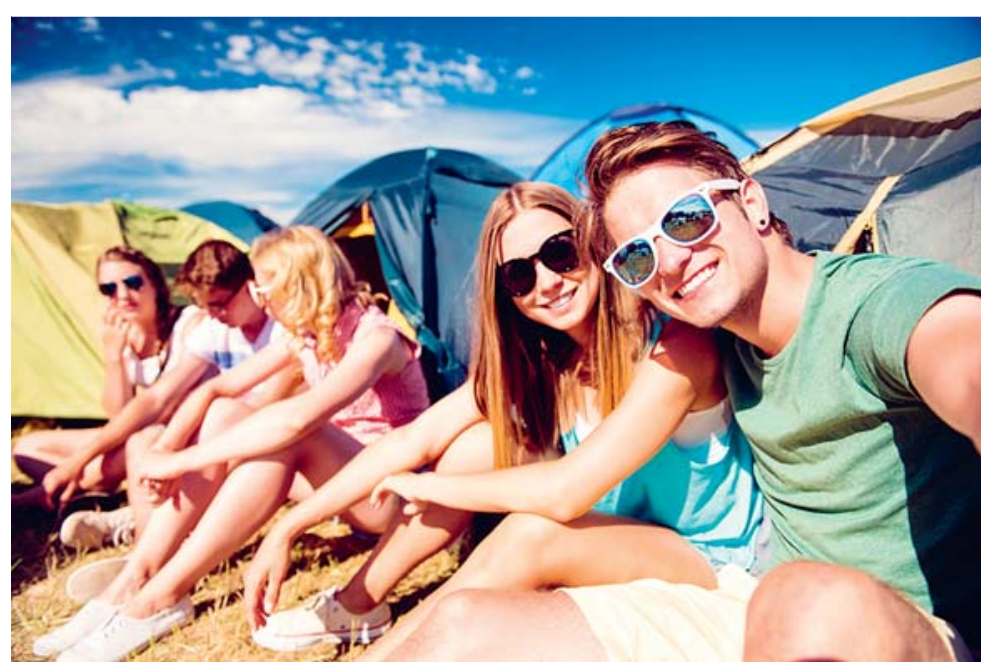

Abb.4 Aufgabe in der Adoleszenz: sich positionieren in der Peer Group. Symbolbild, Quelle: Fotolia. 
Merke: Die Adoleszenz ist eine der chancenreichsten und veränderungsintensivsten Zeiten in der biografischen Entwicklung eines Menschen.

So wird auch deutlich, wie wesentlich die Verfügbarkeit und die Nutzung der idealerweise früh in der Kindheit erworbenen Erfahrungen und Fähigkeiten ist (s.o.). Mit ihnen gelingt es einem Jugendlichen besser, sich den Anforderungen zu stellen, die körperliche und mentale Reifung einzuordnen und die sich weiter herausbildenden Steuerungsfunktionen zu nutzen. Sie stellen auch eine Grundlage dafür dar, die notwendigen und dennoch nicht minder schwierigen Übergänge bei Klassen- und Schulwechseln, den Umgang mit ersten romantischen Gefühlen, Veränderungen der Bezugspartner unter den Gleichaltrigen und den Erwachsenen zu bewältigen und nach Möglichkeit für sich nutzbar zu machen. Gleichfalls wird deutlich, wie schwerwiegend diese Reifungsanforderungen sind, in welche (temporäre) Verzweiflung sie Jugendliche bringen können und welche Auslenkung sie im Rahmen psychosomatischer Probleme mit sich bringen können.

\section{Das Stress-Paradigma}

In der neurobiologischen Forschung wachsen stetig Erkenntnisse, welche jene psychosomatischen Störungskonzepte untermauern, die auf dem „StressParadigma“ basieren. Stresserleben ist erforderlich, um Stressbewältigung zu erlernen. Dabei hat sich erwiesen, dass sich leichte bis mittelgradige Irritationen und Belastungen gut eignen, um Anpassungsleistungen zu befördern, das Lernen zu unterstützen und letztlich eine positive Bewältigung mit sich zu bringen: Eine schwierige Aufgabe wurde gemeistert, und dies wirkt

\section{Natürliche Anforderungen der Adoleszenzentwicklung}

- Lernen, mit dem eigenen Körperbild und seinen Veränderungen fertig zu werden,

- Gewinnen von Selbstvertrauen,

- eigene Fähigkeiten entdecken und nutzen lernen,

- angemessene Ablösung von dem Elternhaus,

- Meistern von Schwellensituationen in der Schule und in persönlichen Beziehungen,

- neue Beziehungen zu Altersgenossen herstellen, sowie Selek- tion und Zurechtkommen mit ihnen,

- einen angemessenen Umgang mit der eigenen Sexualität erlernen,

- Orientierung an einem sich entwickelnden Wertesystem in einer wertepluralen Gesellschaft,

- Begegnung mit einer wettbewerbsorientierten Gesellschaft und hohen Leistungsanforderungen,

- Reflektionen zu sozialer Geltung und sozioökonomischem Status. sich positiv auf das Selbstwirksamkeitserleben sowie den wahrgenommenen Selbstwert aus (Abb.5).

Merke: Wird das System zur Stressbewältigung jedoch durch unangemessen heftige, zu starke oder gar traumatisierende Belastungen überfordert, kann sich eine Schädigung des Stresssystems einstellen.

Grund zu dieser Annahme geben Forschungsergebnisse, die zeigen konnten, dass eine Dauerstimulierung der sympathikotonen Erregung mit einer dauerhaft hohen Kortisolausschüttung zu einer Schädigung der Hippokampusformation führt. In der Folge kommt es zu einer anhaltenden und automatisierten Stimulierung der Amygdala, welche aufgrund der Abkopplung von den reflektierenden und steuernden Einflüssen der kortikalen Zentren eine Beruhigung innerer Zustände kaum noch ermöglicht.

\section{Psychoneuroimmunologie}

Es ist naheliegend, dass ein dauerhaftes Stresserleben nicht nur toxisch für die Entwicklung und Nutzung der Erinnerungsfähigkeit, für die Aufmerksamkeitskontrolle und Lernprozesse im Allgemeinen, sondern offenbar auch für die Gesundheit des gesamten Organismus ist.

Die Psychoneuroimmunologie befasst sich mit den endokrinen und immunologischen Zusammenhängen zwischen psychischen und körperlichen Zuständen und Empfindungen. Dabei werden unter anderem Zusammenhänge und Folgen einer psychischen Dauerbelastung erforscht. Auch hier lässt sich offenbar feststellen, dass eine beständige Ausschüttung von Stresshormonen einen wesentlichen Einfluss auf die Immunregulation hat: Das im Rahmen der Stressreaktion aus der Nebennierenrinde ausgeschüttete Kortisol hat unter anderem die Funktion, Stress-getriggerte proinflammatorische Zytokine der TH-HelferzellenImmunität (z. B. IL-1, IL-6, TNF- $\alpha$ ) zurückzuregulieren.

Ist aber aufgrund von chronisch entzündlichen Prozessen oder aufgrund o.g. chronischer psychischer und psychosozialer Belastung ein beständiger Hyperkortisolismus zu verzeichnen, so nimmt diese Regulationsfähigkeit ab. Das Stress-System reagiert nicht ausreichend auf ankommende Stress- und Immunanreize (Hypokortisolismus), oder es besteht eine zu geringe Antwortbereitschaft auf das Kortisol (Kortikoidresistenz). Dadurch ist der Organismus zu wenig vor über- 
schießenden oder zu lang anhaltenden Immunreaktionen geschützt, die langfristig in einen Zustand leicht erhöhter Entzündungsaktivität münden, der wiederum ausgeprägte gesundheitliche Folgen hat, bis hin zu beschleunigtem Altern und Lebenszeitverkürzung [8].

Erfreulicherweise scheinen solche pathologischen Veränderungen reversibel zu sein. So konnte gezeigt werden, dass erfolgreiche Psychotherapie auch zu einer grundlegenden Korrektur dieser dysfunktionalen physiologischen Muster führt und damit zur Heilung der psychosomatischen Erkrankung beiträgt [9].

\section{Biopsychosoziales Krankheitsmodell}

Chronische psychosoziale Stressoren und unzureichende Fähigkeiten der Stressbewältigung sind an de Entstehung und am Verlauf von somatischen Krankheiten, wie viralen Infektionserkrankungen, koronaren Herzkrankheiten, rheumatischen und atopischen Erkrankungen, sowie gastrointestinalen Erkrankungen beteiligt. Damit wird klar, dass nicht nur bei psychosomatischen Erkrankungen im engeren Sinne, sondern auch bei körperlichen Erkrankungen im Allgemeinen psychischen Prozessen eine erhebliche Bedeutung zukommt. Dies schlägt sich nieder in einem modernen biopsychosozialen Krankheitsverständnis (Abb. 6) und im Bemühen um „ganzheitliche" Behandlungskonzepte.

\section{Definition, Klassifikation und klinisches Erscheinungsbild}

Merke: Unter somatoformen Erkrankungen verstehen wir chronische körperliche Beschwerden, für die sich keine ausreichenden organischen Ursachen finden lassen, oder bei denen eine auf körperliche Symptome bezogene, rein medizinische Behandlung nicht zu einer ausreichenden Besserung der Beschwerden und Beruhigung des Patienten führt.

Sowohl Erkrankungen, die in den Bereich der Dissoziativen Störungen, als auch solche, die in den Bereich der Somatoformen Störungen nach ICD-10 fallen, zählen zu den psychosomatischen Erkrankungen. Während sich die Dissoziativen Störungen auf Funktionseinschränkungen oder -ausfälle von Motorik und Sensorik oder auch auf nichtepileptische psychogene Anfälle beziehen, stehen bei den Somatoformen Störungen Schmerzen sowie Beschwerden im Bereich des vegetativen Systems im Vordergrund. Beide Störungsgruppen

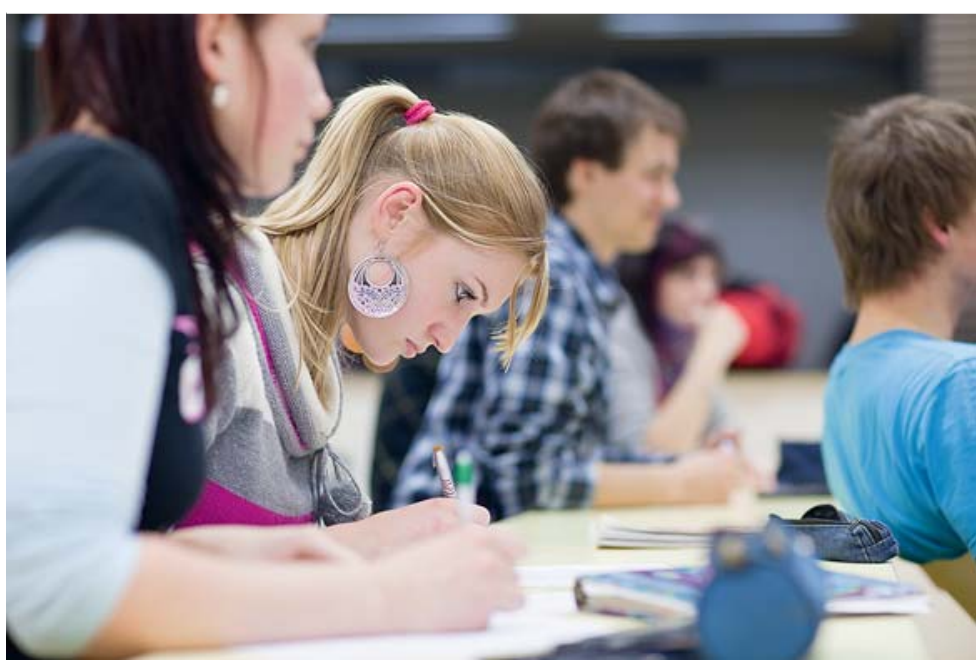

Abb. 5 Stresserleben ist erforderlich, um Stressbewältigung zu erlernen - mit leichten bis mittelgradigen Belastungen. Symbolbild, Quelle: Thieme Verlagsgruppe.

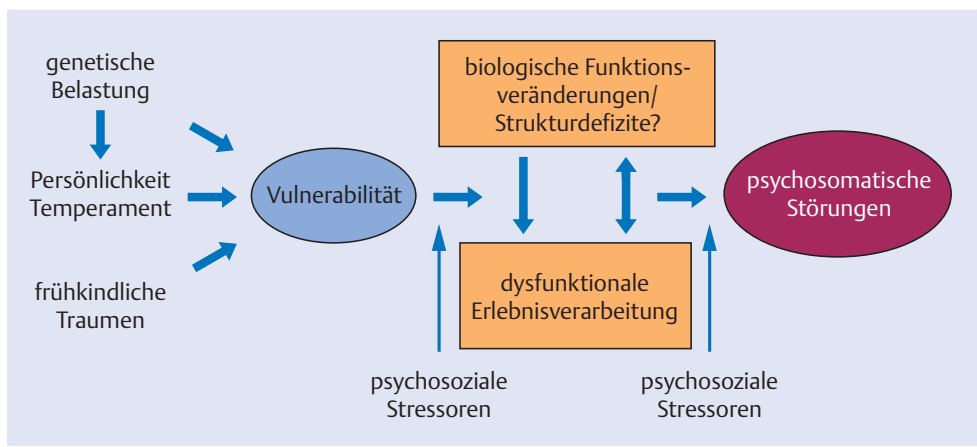

Abb. 6 Biopsychosoziales Krankheitsmodell. Aus: Möller H.-J., Laux G. Duale Reihe Psychiatrie, Psychosomatik und Psychotherapie. 5. Aufl. 2013; Georg Thieme Verlag, Stuttgart.

zeichnen sich jedoch durch eine fehlende Passung zwischen den subjektiv erlebten Beschwerden des Patienten und den (unzureichenden) somatischen Befunden des Kinder- oder Allgemeinarztes aus.

Ein weiteres Missverhältnis besteht häufig zwischen der angegebenen Intensität der Beeinträchtigung (z.B. durch Angabe eines sehr hohen Wertes auf der Schmerzskala) und der Art, wie diese Beeinträchtigung hervorgebracht wird. Massive Beschwerden oder Einschränkungen können indifferent oder sogar mit einem Lächeln präsentiert werden (sog. belle indifférence). Das Ernstnehmen der geschilderten Beschwerden durch den Arzt kann durch dieses Phänomen erheblich erschwert werden und zu Verunsicherung auf allen Seiten führen. Andererseits besteht die Gefahr, maskierte psychische Symptome zu übersehen, weil sich keine offensichtlichen Hinweise etwa auf eine depressive Verstimmung oder eine Angsterkrankung erkennen lassen. Auch bei Patienten und Eltern stellt sich so 


\section{Geschichtliche Einordnung}

Sigmund Freud war vermutlich der Erste, der eine Unterscheidung zwischen psychischen und psychosomatischen Erkrankungen vornahm, auch wenn er selbst den Begriff der Psychosomatik nicht benutzte. Freud prägte den Begriff der Konversion, der auch heute noch für eine Unterform der Dissoziativen Störungen gebräuchlich ist (auch wenn sich das Erscheinungsbild im Laufe der Zeit deutlich verändert hat). Er formulierte als erster, dass es „unverträgliche Vorstellungen“ gebe, die „in Körperliches“ umgesetzt werden. In den 1950er Jahren wurden die folgenden Erkrankungen durch Franz Alexander als psychosoma- tisch beschrieben: peptisches Magengeschwür, ulzerative Kolitis, Bronchialasthma, Thyreotoxikose, essentielle Hypertonie, rheumatoide Arthritis und Neurodermitis. Er entwickelte die Idee, dass Patienten, die an diesen Erkrankungen leiden, passiv-abhängige Persönlichkeitsmerkmale aufweisen und ihre unbewussten Konflikte in den jeweiligen Erkrankungen zum Ausdruck bringen.

Alle diese Erkrankungen können inzwischen pathophysiologisch erklärt werden, wohingegen ein ätiologischer Bezug zu bestimmten Persönlichkeitstypen bis heute nicht eindeutig herstellbar ist.

schnell ein Gefühl der Ohnmacht ein. Folgen sind hartnäckige Forderungen nach Untersuchungen und häufig auch ein aufmerksamkeitssuchendes Verhalten der Patienten.

Im Kindes- und Jugendalter gibt es auch die Möglichkeit, dass sich die Eltern übermäßig Sorgen um die Gesundheit ihrer Kinder machen und auf weitere Untersuchungen drängen. Eine solche Fehlanpassung bei Eltern wird bei besonderer Ausprägung nach Noeker (2008) als Somatoforme Störung by Proxy (durch Stellvertreter) definiert [10].

\section{Den Beginn der Symptomatik sorgfältig explorieren}

Meistens können bei sorgfältiger Exploration nahe zurückliegende Belastungen als Auslöser herausgearbeitet werden. Im Kindesalter sind dies vorrangig familiäre und/oder schulische Belastungen, während im Jugendalter meist innere Konflikte die Bewältigung erforderlicher Entwicklungsschritte behindern.

Bei den Dissoziativen Störungen können einfache körperliche Erkrankungen oder Ereignisse den psychosomatischen Krankheitsprozess auslösen, der dann für den ausbleibenden Gesundungsprozess und die durch somatische Befunde nicht erklärbare Funktionsbeeinträchtigung verantwortlich ist. Insgesamt finden sich bei Patienten mit psychosomatischen Erkrankungen

bzw. in deren Familien gehäuft psychosoziale Belastungsfaktoren und dysfunktionale Kommunikationsmuster - oftmals über mehrere Generationen hinweg. Diese Zusammenhänge können jedoch meist weder die Patienten selbst noch die Eltern erkennen oder benennen: Sie werden bewusstseinsfern wirksam. Eine zu schnelle Konfrontation mit dem Verdacht einer psychischen Ursache oder einer psychiatrischen Erkrankung kann daher leicht abgewehrt und als Beleidigung, Bagatellisierung und Bedrohung aufgefasst werden.

Eine eingehende Anamnese zu Gesundheitsproblemen der Eltern und engerer Verwandter sollte erhoben werden. Gehäuft finden sich bei den Eltern bis in die eigene Jugend zurückreichende ähnliche Schmerzoder Beeinträchtigungssymptome und aktuelle psychiatrische Erkrankungen sowie psychosoziale Belastungen. Auch „Eintrittspforten“ sind gehäuft zu finden, das heißt, das Kind (oder eben ein Elternteil) war vor Wochen oder Monaten somatisch mit einer ähnlichen Symptomatik erkrankt.

\section{Dissoziative Störungen}

Störungen, die früher als Konversionsstörungen (s. Geschichtliche Einordnung; vgl. auch [11,12]) oder Hysterie klassifiziert wurden, finden sich heute in der ICD 10 unter dem Begriff Dissoziative Störungen (Tab.1). Dissoziative Phänomene sind im Alltag häufig als gesunde Übergangsphänomene wahrnehmbar, beispielsweise die empfundene „Loslösung“ vom Körper beim Joggen. Patienten mit Dissoziativen Störungen verlieren dauerhaft die Fähigkeit, ihre Erinnerungen, ihre sensorischen Empfindungen oder Körperbewegungen in ihr Selbstbild zu integrieren.

Merke: Dissoziative Störungen haben oft eine enge zeitliche Verbindung mit erlebten Traumata, unlösbaren, unerträglichen Konflikten oder gestörten Beziehungen.

Die dissoziativen Sensibilitäts- und Bewegungsstörungen stellen sich häufig eher so dar, wie Patienten sich vorstellen, dass die Erkrankung aussehen müsste, z.B. strumpfförmige, statt Dermatom-angepasste Sensibilitätsstörungen. Wichtige Hinweise auf eine Dissoziative Störung sind: plötzlicher Beginn, Variabilität der präsentierten Symptomatik (z. B. Veränderungen der Tremorfrequenz oder der Sensibilitätsgrenzen in verschiedenen Untersuchungen), intermittierende Symptomatik, andere somatoforme Störungen in der Vorgeschichte, Hinweise auf Traumatisierungen und ein 
Tabelle 1

Dissoziative Störungen nach ICD-10.

\begin{tabular}{|c|c|c|c|}
\hline Schlüssel & Diagnose & Kriterien und Besonderheiten & Vorkommen im Kindes- und Jugendalter \\
\hline F44.0 & Dissoziative Amnesie & $\begin{array}{l}\text { Verlust der Erinnerung an wichtige aktuelle Ereignisse, } \\
\text { selektiv und unvollständig. Nicht bei hirnorganischen } \\
\text { Störungen, Intoxikationen, extremer Erschöpfung. Geht } \\
\text { jedoch deutlich über „normale“ Vergesslichkeit, z. B. im } \\
\text { Rahmen von Müdigkeit, hinaus. }\end{array}$ & $\begin{array}{l}\text { häufiger; meist auf traumatische Ereignisse } \\
\text { bezogen }\end{array}$ \\
\hline F44.1 & Dissoziative Fugue & $\begin{array}{l}\text { Zielgerichtete Ortsveränderung mit dissoziativer Amne- } \\
\text { sie, häufig vollständig und generalisiert. Das Verhalten } \\
\text { kann dennoch von außen völlig normal wirken. }\end{array}$ & $\begin{array}{l}\text { seltener; häufiger nach schweren (z. B. sexuel- } \\
\text { len) Traumata im Sinne einer Reinszenierung } \\
\text { mit ungewolltem/unbewusstem Wiederauf- } \\
\text { suchen des Täters }\end{array}$ \\
\hline F44.2 & Dissoziativer Stupor & $\begin{array}{l}\text { Deutliche Verringerung oder Fehlen von Reaktionen auf } \\
\text { Reize (Licht, Berührung, Geräusche) und Fehlen von will- } \\
\text { kürlichen Bewegungen. Kein Anhalt für eine körperliche } \\
\text { Ursache. Nicht im Rahmen anderer Selten psychiatri- } \\
\text { scher Erkrankungen (Depressionen, Katatonie etc.). }\end{array}$ & selten \\
\hline F44.3 & $\begin{array}{l}\text { Trance und Besessen- } \\
\text { heitszustände }\end{array}$ & $\begin{array}{l}\text { Verlust der Identität und der Wahrnehmung der Umge- } \\
\text { bung (unfreiwillig, ungewollt, nicht religiös oder kulturell } \\
\text { akzeptiert ausgelöst) }\end{array}$ & selten \\
\hline F44.4 & $\begin{array}{l}\text { Dissoziative Bewegungs- } \\
\text { störung }\end{array}$ & $\begin{array}{l}\text { Vollständiger oder teilweiser Verlust der Bewegungs- } \\
\text { fähigkeit eines oder mehrerer Körperglieder (inkl. Apho- } \\
\text { nie und Dysphonie). Das klinische Bild imponiert wie eine } \\
\text { neurologische Bewegungsstörungen, z. B. Ataxie, Dyski- } \\
\text { nesie, Lähmung. }\end{array}$ & häufiger \\
\hline F44.5 & Dissoziative Krampfanfälle & $\begin{array}{l}\text { Klinisches Bild ähnlich den Bewegungen bei epilepti- } \\
\text { schen Anfällen, jedoch seltener Verletzungen beim Sturz, } \\
\text { fast nie Zungenbiss, fast nie Inkontinenz. Ein Bewusst- } \\
\text { seinsverlust fehlt, eher stuporähnliche Zustände. }\end{array}$ & $\begin{array}{l}\text { häufiger; auch als Mischform mit epileptischen } \\
\text { Anfällen }\end{array}$ \\
\hline F44.6 & $\begin{array}{l}\text { Dissoziative Sensibilitäts- } \\
\text { und Empfindungsstörungen }\end{array}$ & $\begin{array}{l}\text { Unterschiedliche Ausfälle sensorischer Modalitäten, } \\
\text { Grenzen eher laienhaft, häufig von Parästhesien beglei- } \\
\text { tet, auch Seh- oder Hörstörungen können vorkommen. }\end{array}$ & seltener \\
\hline F44.7 & $\begin{array}{l}\text { Dissoziative Störungen, } \\
\text { gemischt }\end{array}$ & Kombination der oben genannten & selten \\
\hline F44.8 & $\begin{array}{l}\text { Sonstige Dissoziative } \\
\text { Störungen }\end{array}$ & $\begin{array}{l}\text { Ganser-Syndrom, Multiple Persönlichkeitsstörung, } \\
\text { Transitorische dissoziative Störungen in der Kindheit } \\
\text { und Jugend und inkl. psychogenem Dämmerzustand, } \\
\text { Verwirrtheit }\end{array}$ & häufiger \\
\hline F44.9 & $\begin{array}{l}\text { Nicht näher bezeichnete } \\
\text { Dissoziative Störungen }\end{array}$ & Restkategorie, nicht definiert & - \\
\hline
\end{tabular}


Abb. 7 Bei Kindern mit Somatoformen Störungen sind am häufigsten Kopfoder Bauchschmerzen zu finden. Symbolbild, Quelle: Fotolia.

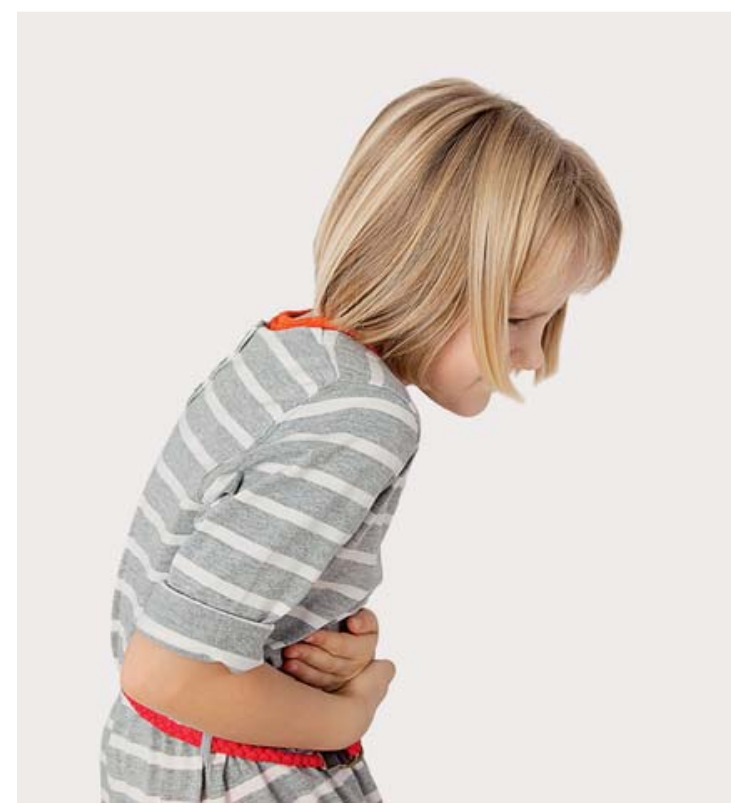

sekundärer Krankheitsgewinn, wohingegen etwa Verletzungen infolge von psychogenen Anfällen fast nie auftreten. Angst und Depression sind häufig komorbid vorhanden, jedoch nicht deutlich mehr als in der Normalbevölkerung.

\section{Somatoforme Störungen}

Kennzeichnend sind hier körperliche Symptome, insbesondere Schmerzen oder vegetative Beschwerden, die nicht durch krankhafte Organbefunde begründbar sind (Tab. 2).

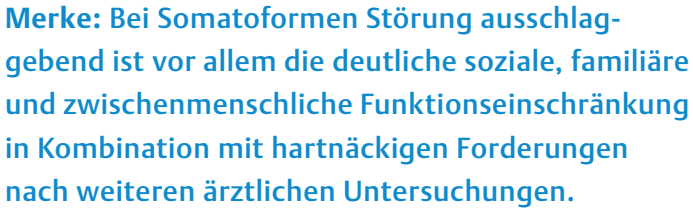

Merke: Bei Somatoformen Störung ausschlaggebend ist vor allem die deutliche soziale, familiäre und zwischenmenschliche Funktionseinschränkung in Kombination mit hartnäckigen Forderungen nach weiteren ärztlichen Untersuchungen.

Eine altersspezifische Einteilung existiert bis heute nicht, daher fehlen aus kinderpsychiatrischer Sicht auch spezifische Kriterien für die Somatoformen Störungen des Kindes- und Jugendalters. Im Vorschulalter sind Somatoforme Störungen eher selten, und im Grundschulalter finden sich kürzere Verläufe und monosymptomatische Erscheinungsbilder. Am häufigsten sind Kopf- und Bauchschmerzen zu finden (Abb.7) [13]. Der Erkrankungswert ergibt sich häufig aus den raschen Einschränkungen im Alltag, z. B. mit gehäuften Fehlzeiten in der Schule und sozialem Rückzug.
Im Pubertätsalter nähert sich das Erscheinungsbild dem des Erwachsenenalters an. Die Symptomatik ist häufig vielfältiger und die Symptome bestehen über Monate oder Jahre; lang anhaltende Belastungen und langfristige Schulversäumnis sind die Folge. Bei nicht adäquater Behandlung droht eine Chronifizierung, die weit bis ins Erwachsenenalter andauern kann.

\section{Diagnose und Differenzial- diagnose}

\section{Diagnostische Hilfsmittel}

Die Diagnosestellung bei psychosomatischen Erkrankungen erfolgt klinisch nach definierten Kriterien.

Im deutschsprachigen Raum existieren kaum spezifische Instrumente zur Erfassung von somatoformen Störungen. Für das Jugendalter ab 16 Jahren, gibt es ein Computer-gestütztes Interview (CIDI/DIA-X), welches die Diagnose nach ICD-10 und DSM-IV bestätigen kann, es ist als Screeningverfahren jedoch nicht geeignet. Der Gießener Beschwerdebogen für Kinder und Jugendliche (GBB-KJ) enthält eine Liste körperlicher Symptome, jedoch ohne die sonstigen Diagnosekriterien zu berücksichtigen.

Fragen zur Erfassung

somatoformer Störungen

Für eine grobe subjektive Orientierung in der Praxis empfiehlt es sich nach Noeker (2008), die folgenden Fragen auf einer Skala von 1 (sehr wenig) bis 6 (sehr stark) einschätzen zu lassen [14]:

Auf Elternseite:

- Wie stark fühlt sich der Schmerz für Ihr Kind an?

- Wie stark ist ihre Angst vor einer ernsthaften Krankheit Ihres Kindes?

- Wie stark hält der Schmerz ihr Kind davon ab, bestimmte Dinge zu tun (Freunde treffen, Schule, Hobbies, Familie)?

\section{Auf Kindseite:}

- Wie stark fühlt sich der Schmerz in deinem Körper an?

- Wie stark ist Deine Angst vor einer schlimmen Krankheit?

- Wie stark hält Dich Dein Schmerz davon ab, bestimmte Dinge zu tun (Freunde treffen, Schule, Hobbies, Familie)? 
Tabelle 2

Somatoforme Störungen nach ICD-10.

\begin{tabular}{|c|c|c|c|}
\hline Schlüssel & Diagnose & Kriterien und Besonderheiten & $\begin{array}{l}\text { Vorkommen im Kindes- } \\
\text { und Jugendalter }\end{array}$ \\
\hline F45.0 & Somatisierungsstörung & $\begin{array}{l}\text { Mindestens } 2 \text { Jahre bestehend mit multiplen körperlichen Sympto- } \\
\text { men. Können jedes Körperteil und jedes Körpersystem betreffen. } \\
\text { Häufig chronisch, fluktuierend. Mindestens } 6 \text { Symptome aus } 2 \text { ver- } \\
\text { schieden Kategorien (gastrointestinal, kardiovaskulär, urogenital, } \\
\text { Haut und Schmerzsymptome). Häufige „Patienten-Karriere“ mit } \\
\text { häufigen, explorativen Operationen. }\end{array}$ & $\begin{array}{l}\text { weniger bei Kindern, eher ab dem } \\
\text { Jugendalter }\end{array}$ \\
\hline F45.1 & $\begin{array}{l}\text { Undifferenzierte } \\
\text { Somatisierungsstörung }\end{array}$ & $\begin{array}{l}\text { Weniger als } 2 \text { Jahre oder weniger auffallende Symptomatik. Es } \\
\text { liegen auch hier verschiedene Beschwerden vor, das typische Bild, } \\
\text { oder das Zeitkriterium sind aber nicht erfüllt. }\end{array}$ & $\begin{array}{l}\text { insbesondere aufgrund der Zeit- } \\
\text { dauer häufiger im Kindes- und } \\
\text { Jugendalter diagnostiziert }\end{array}$ \\
\hline F45.2 & Hypochondrische Störung & $\begin{array}{l}\text { Dauerhafte Beschäftigung mit der Möglichkeit zu erkranken, oder } \\
\text { der Überzeugung bereits erkrankt zu sein. Häufig anhaltende kör- } \\
\text { perliche Beschwerden oder ständige Beschäftigung mit der eigenen } \\
\text { körperlichen Erscheinung im Sinne einer Angst vor Entstellung } \\
\text { (Dysmorphophobie) oder einer nicht wahnhaften Überzeugtheit, } \\
\text { entstellt zu sein. Normale Körperreaktionen werden als abnorm, } \\
\text { belastend interpretiert. Häufig in Kombination mit Depression oder } \\
\text { Angsterkrankungen. }\end{array}$ & häufiger ab dem Jugendalter \\
\hline F45.3 & $\begin{array}{l}\text { Somatoforme autonome } \\
\text { Funktionsstörung }\end{array}$ & $\begin{array}{l}\text { Vegetativ innervierte Organsysteme mit subjektiven Beschwerden: } \\
\text { Schmerzen, Brennen, Enge, Schwere. Die Patienten sind der Über- } \\
\text { zeugung, dieses Organsystem sei erkrankt (kardiovaskulär [F45.30\}, } \\
\text { gastrointestinal [F45.31/F45.32\}, respiratorisch [F45.33\}, urogeni- } \\
\text { tal \{F54.34\}). Fast immer in Kombination mit vegetativ vermittelten } \\
\text { Angstsymptomen wie Herzklopfen, Zittern, Schwitzen. }\end{array}$ & häufiger ab dem Jugendalter \\
\hline F45.40 & $\begin{array}{l}\text { Anhaltende Schmerz- } \\
\text { störung }\end{array}$ & $\begin{array}{l}\text { Andauernder, schwerer, quälender Schmerz, der nicht oder nicht } \\
\text { hinreichend durch physiologische Befunde erklärt werden kann. } \\
\text { Fast immer in Verbindung mit Konflikten, Belastungen (beeinflus- } \\
\text { sen Beginn, Schweregrad, Exazerbation, Aufrechterhaltung), fast } \\
\text { immer gesteigerte Hilfen (medizinisch, persönlich). Darf nicht im } \\
\text { Verlauf anderer psychiatrischer Erkrankungen wie Depressionen } \\
\text { oder Schizophrenie diagnostiziert werden. }\end{array}$ & häufiger \\
\hline F45.41 & $\begin{array}{l}\text { Chronische Schmerzstörung } \\
\text { mit somatischen und } \\
\text { psychischen Faktoren }\end{array}$ & $\begin{array}{l}\text { Mindestens } 6 \text { Monate bestehende Schmerzen, die ihren Ursprung in } \\
\text { einer körperlichen Erkrankung haben. Der Beginn ist also durch } \\
\text { pathologische Organbefunde begründet, dagegen sind Aufrecht- } \\
\text { erhaltung, Schweregrad, Exazerbation psychisch bedingt. }\end{array}$ & $\begin{array}{l}\text { vor allem bei chronisch erkrankten } \\
\text { Kindern- und Jugendlichen }\end{array}$ \\
\hline F45.8 & Sonstige Schmerzstörung & $\begin{array}{l}\text { Störungen der Wahrnehmung von Körperfunktionen, die nicht } \\
\text { vegetativ vermittelt werden, wie psychogene Dysmenorrhoe, } \\
\text { Dysphagie, Pruritus, Tortikollis, Zähneknirschen }\end{array}$ & seltener \\
\hline F45.9 & $\begin{array}{l}\text { Nicht näher bezeichnete } \\
\text { Schmerzstörung }\end{array}$ & undefinierte Restkategorie & - \\
\hline
\end{tabular}


Tabelle 3

Psychiatrisch relevante Differenzialdiagnosen.

\begin{tabular}{|c|c|}
\hline Differenzialdiagnose & Kriterien \\
\hline $\begin{array}{l}\text { psychologische Faktoren und Ver- } \\
\text { haltensfaktoren bei anderenorts } \\
\text { klassifizierten Krankheiten }\end{array}$ & $\begin{array}{l}\text { dient der Erfassung von psychologischen } \\
\text { Faktoren, die den Verlauf bzw. die Behand- } \\
\text { lungscompliance bei bestehenden körper- } \\
\text { lichen Erkrankungen beeinflussen (z. B. } \\
\text { Asthma, Diabetes mellitus) }\end{array}$ \\
\hline Depressionen & $\begin{array}{l}\text { häufig begleitende somatische Beschwerden, } \\
\text { jedoch auch deutlich gedrückte Stimmung, } \\
\text { Freudverlust, Antriebsmangel }\end{array}$ \\
\hline $\begin{array}{l}\text { Panikstörung und generalisierte } \\
\text { Angststörung }\end{array}$ & $\begin{array}{l}\text { vegetativ vermittelte Anzeichen von Angst, } \\
\text { die als Erkrankung fehlinterpretiert werden } \\
\text { können, jedoch häufiger episodenhaftes Auf- } \\
\text { treten und breiteres Spektrum an Dingen, } \\
\text { über die sich Sorgen gemacht wird }\end{array}$ \\
\hline $\begin{array}{l}\text { Depersonalisierungs- und } \\
\text { Derealisationsphänomene }\end{array}$ & $\begin{array}{l}\text { im Rahmen starker psychischer Belastungen } \\
\text { (z. B. akute Traumaverarbeitung oder auch } \\
\text { bei Drogenintoxikation) erlebte Distanzie- } \\
\text { rung vom eigenen Körper oder der Umge- } \\
\text { bung }\end{array}$ \\
\hline Psychosen & $\begin{array}{l}\text { im Rahmen von Wahnsystemen auftretende } \\
\text { Erkrankungsängste, dabei jedoch auch } \\
\text { weitere psychotische Symptome }\end{array}$ \\
\hline $\begin{array}{l}\text { Münchhausen by Proxy/Kindes- } \\
\text { misshandlung }\end{array}$ & $\begin{array}{l}\text { durch Eltern herbeigeführte Symptome mit } \\
\text { Schädigung des Kindes }\end{array}$ \\
\hline $\begin{array}{l}\text { artifizielle Störung (absichtliches } \\
\text { Erzeugen oder Vortäuschen von } \\
\text { körperlichen oder psychischen } \\
\text { Symptomen oder Behinderungen) }\end{array}$ & $\begin{array}{l}\text { Verhaltensweisen/Selbstverletzungen, mit } \\
\text { denen Symptome erzeugt werden oder vor- } \\
\text { getäuscht werden; keine äußere Motivation } \\
\text { (z. B. finanzielle Entschädigung, Flucht vor } \\
\text { Gefahr, mehr medizinische Versorgung); } \\
\text { Ausschluss einer erklärenden körperlichen } \\
\text { oder psychischen Störung }\end{array}$ \\
\hline Simulation & $\begin{array}{l}\text { vorgetäuschte Krankheit mit deutlicher, } \\
\text { dahinter liegender Motivation }\end{array}$ \\
\hline $\begin{array}{l}\text { Entwicklung körperlicher Symp- } \\
\text { tome aus psychischen Gründen }\end{array}$ & $\begin{array}{l}\text { Aggravierung oder längeres Anhalten von } \\
\text { körperlichen Symptomen, die ursprünglich } \\
\text { durch körperliche Störung erklärt werden } \\
\text { konnten; Vorliegen von Hinweisen auf psy- } \\
\text { chische Verursachung (Enttäuschung über } \\
\text { mangelhafte Betreuung, Angst vor Behin- } \\
\text { derung etc.) }\end{array}$ \\
\hline
\end{tabular}

\section{Differenzialdiagnose}

Im Rahmen diagnostischer Erwägungen muss immer das Vorliegen anderer psychiatrischer Erkrankungen in Betracht gezogen werden. Tab. 3 gibt eine Übersicht über mögliche Differenzialdiagnosen.

\section{Schlussfolgerungen für die kinderärztliche Praxis}

\section{Anamnese und Gesprächsführung}

\author{
Merke: Eine sorgfältige, auch über die unmittel- \\ baren Beschwerdesymptome hinausgehende \\ Exploration der Gesundheits-, Sozial- und Familien- \\ anamnese ist unabdingbar.
}

Mittelfristig lassen sich so Zeit und Doppeluntersuchungen sparen, und ein besserer Überblick über die Gesamtproblematik ist herzustellen. Der weit verbreitete Gedanke „Besser nicht genau fragen, weil ich ihn/ sie dann noch auf Ideen bringen könnte“, ist an dieser Stelle unangebracht.

Die Gesprächstechniken, die in diesem Zusammenhang zielführend sind, umfassen vor allem eine Haltung des „Annehmens“ der Beschwerden. Hilfreich sind offene Fragen wie:

- Erzähl mal, wie sind die Beschwerden?

- Kannst Du sie mir genauer beschreiben?

- Was denkst Du selbst über diese Beschwerden?

- Ärgerst Du Dich, wenn diese Beschwerden auftreten?

Dabei ist es wichtig, immer den Kontext der Beschwerden genauer zu erfragen:

- Gibt es spezielle Situationen, in denen die Beschwerden stärker sind?

- Welche Folgen hat das für Deine Freundschaften, für die Familie?

- Wer kümmert sich in solchen Situationen um Dich?

- Was hilft Dir bei diesen Beschwerden?

Kommt der Arzt dem Wunsch des Patienten nach weiteren Untersuchungen nach, so wird sich an der Symptomatik nichts ändern und das Konzept der körperlichen Krankheit weiter zementiert. Verweigert er sich diesem Wunsch aus dem Gefühl heraus, manipuliert zu werden, entstehen ablehnende und negative Gefühle dem Patienten gegenüber, der Kontakt wird distanziert oder gar abgebrochen. Eine zu drastische Deutung der Beschwerden als Ausdruck psychischer Probleme wird 


\section{Wie sag ich es meinem Patienten? - Beispiele für eine hilfreiche Kommunikation [14]}

1. „Im Darm sind besonders viele Nervenzellen, die bei Ihrem Kind schon auf kleinste Reizungen wie z. B. normale Darmbewegungen mit Schmerzen reagieren können. Diese hoch empfindsamen Nervenzellen des Darmes stehen in enger Verbindung zum Gehirn und können daher mitreagieren, wenn Ihr Kind sich aufregt oder Sorgen hat. Dies gilt besonders bei Kummer, den Ihr Kind nicht mit Worten ausdrücken kann. Manche Kinder oder Eltern verwechseln dann Anzeichen seelischer Aufregung mit körperlicher Krankheit.“

2. „Mindestens ebenso wichtig ist es, negative Auswirkungen der wiederkehrenden Bauchschmerzen auf die normale Entwicklung des Kindes in Familie, Schule und Freundeskreis in Grenzen zu halten. Dazu kann begleitend zur ärztlichen Behandlung auch eine psychologische Beratung hilfreich sein."
3. Ein anschauliches Video zur Erklärung von Schmerzstörungen mit dem Titel „Den Schmerz verstehen - und was zu tun ist in 10 Minuten “ des Deutschen Kinderschmerzzentrums ist für Patienten gut geeignet und findet sich in vielen verschiedenen Sprachen unter http://www.deutsches-kinderschmerzzentrum.de/jugendliche/video-denschmerz-verstehen/ ggf. von Patienten als beleidigend empfunden und zurückgewiesen.

Merke: Ziel erster Gespräche in der kinderärztlichen Praxis ist es, dass sich zwischen dem Patienten bzw. der Familie und dem Arzt ein tragfähiges Bündnis entwickeln kann.

Psychosomatisch erkrankte Patienten erleben ihre Beschwerden tatsächlich und simulieren nicht. Empfinden sie sich jedoch mit ihren Beschwerden nicht gesehen und nicht ernst genommen, vermeintlich auch noch zurückgewiesen, so gibt es zumeist zwei Möglichkeiten: Entweder werden Betroffene mit einer Vielzahl von Symptomen immer wieder versuchen zu überzeugen, oder sie suchen schließlich einen anderen Arzt auf, von dem sie sich mehr Verständnis und Hilfe versprechen.

Gleichwohl ist es wichtig, die Patienten aus ihrer Passivität zu locken und ihre oft übergroßen Erwartungen an körperbezogenes ärztliches Handeln zu mindern. Fragetechniken, die ein Bewusstsein für den Einfluss eigener Ideen und Überzeugungen schaffen, stärken das Gefühl, nicht allem passiv ausgeliefert zu sein, und damit eine Idee von Selbstwirksamkeit. Insbesondere für einen „würdevollen Rückzug aus dem Symptom“, ist es wichtig, auf Hinweise und Ideen von Patienten über das ermutigend einzugehen, was sie selbst meinen, zur Linderung ihrer Beschwerden beitragen zu können.

Ist ein tragfähiges Bündnis zwischen Arzt und Patient erreicht, müssen die Inhalte und Ergebnisse der schon erfolgten und noch erforderlichen Untersuchungen in Ruhe besprochen werden.

Merke: Die Erläuterung der Ergebnisse sollte sich darauf beziehen, dass eine hinreichende Erklärung der Beschwerden nicht mit Hilfe einer körperlichen Diagnostik erfolgen kann und dass dennoch weiterer (psychosomatischer) Behandlungsbedarf besteht.

Für den Patienten ist dies meist Voraussetzung dafür, dass er auch andere Gedanken zulassen kann. In der Folge sollten dann regelmäßigere, von den Beschwerden zunehmend unabhängige Kontakte und Wiedervorstellungen erfolgen, in denen die Zuwendung des Arztes mehr und mehr auf die gesunden Anteile des Patienten und seine Genesungsbemühungen gelenkt werden können. Auf diese Weise wird der Fokus viel mehr auf die gesündere Seite und Genesung gerichtet, als auf eine durch Störungen fokussierte Problematik.

Dieses Vorgehen scheint vor allem dann vielversprechend, wenn die Problematik überschaubar ist, die Symptome nicht multipel sind und das Funktionsniveau des Patienten noch ausreichend gut ist.

\section{Hinzuziehen eines Kinder- und Jugendpsychiaters}
Merke: Sind die Symptome so stark, dass beispiels- weise der Schulbesuch unmöglich ist, so erscheint es ratsam, einen Kinder- und Jugendpsychiater hin- zu zu ziehen. Ideal ist dann zunächst ein gemeinsa- mes Gespräch mit dem behandelnden Kinderarzt, dem Kinderpsychiater und dem Patienten bzw. dessen Eltern.

Ein solches Gespräch kann Missverständnissen vorbeugen. Der Kinderpsychiater wird versuchen, Hintergründe und Einflussfaktoren genauer zu beleuchten und therapeutische Schritte mit dem Betroffenen und dessen Familie zu erarbeiten. Eine psychiatrische Medikation erscheint vorrangig bei komorbiden affek- 


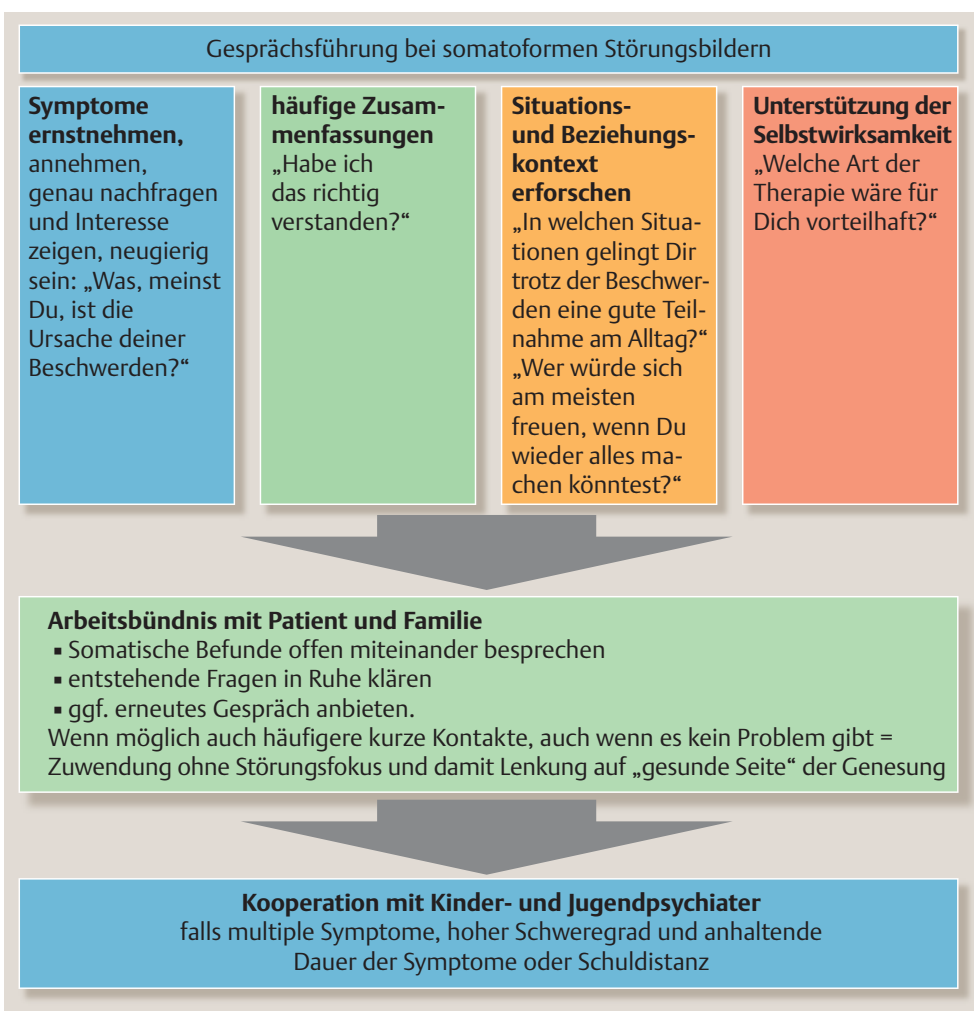

Abb. 8

Haus- bzw. kinderärztliche Versorgung bei somatoformen Störungen.

tiven Störungen indiziert (siehe Abschnitt zur Medikation weiter unten). Für den Kinder- und Jugendpsychiater ist eine psychosomatische Erkrankung keine Ausschlussdiagnose, sondern vielmehr eine eigenständige, an definierten Kriterien zu erfassende Diagnose. Dennoch ist es auch für ihn wichtig, genau zu wissen, welche diagnostischen Schritte bereits unternommen wurden und was die bisherigen Ergebnisse waren.

Die folgende grobe Checkliste kann bei der Vermittlung an einen weiterführenden psychotherapeutisch arbeitenden Kollegen wichtig sein, um die notwendigen Informationen zu erhalten:

Die wichtigsten Elemente für die pädiatrische Versorgung sind als Flowchart in Abb. 8 und zusammengefasst als „Dos and Don'ts“ in Tab. 4 dargestellt.

\section{Checkliste}

Für die Übergabe an einen Kinder- und Jugendpsychiater und Psychotherapeuten wichtig:

- bisherige somatische Befunde: Sind schwerwiegende und lebensbedrohliche Erkrankungen ausgeschlossen worden und wurde dies mitgeteilt?

- Art und Dauer der Symptome - Wie ist das somatische Inanspruchnahmeverhalten?

- Schweregrad der Beeinträchtigung/extremer Leidensdruck? Besteht eine Neigung zur Katastrophisierung bzw. der Verdacht auf eine Hypochondrie?

- Bestehen negative Auswirkungen auf schulische und soziale Entwicklung und auf die Familie, z. B. Schulabsentismus oder Aufgabe von Hobbies?

- Bestehen schon bekannte komorbide psychiatrische Erkrankungen wie Angst, Depression, Persönlichkeitsstörungen?

\section{Fallbeispiele}

\section{Fallbeispiel 1: „Hätte ich die Bauchschmerzen nicht, wäre alles gut.“}

Greta, 8 Jahre, wird im kinderpsychiatrischen Konsil in der Kinderklinik erstmalig vorgestellt. Ihre Eltern berichten, dass die Symptomatik vor einem Jahr mit gelegentlichen Bauchschmerzen begonnen und sich innerhalb dieser Zeit so gesteigert haben, dass Greta inzwischen die Schule nicht mehr besuchen könne. Greta verbringe die Vormittage zuhause, die Mutter sei nun ebenfalls krankgemeldet. Greta schlafe aus, dann schaue sie Fernsehen oder spiele am Tablet-PC; ihre Hobbies, Tanzen und Reiten, habe sie ebenfalls aufgeben müssen. Nachmittags komme der jüngere Bruder nach Hause und die beiden würden gelegentlich miteinander spielen. Greta selbst sagt, dass sie gerne wieder in die Schule gehen würde und mehr Freunde treffen möchte, aber dass dies aufgrund der Bauchschmerzen nicht gehe. Ihre Stimmung sei gut, sie habe keine Angst vor der Schule.

An Voruntersuchungen haben bei verschiedenen Ärzten mehrere Stuhl- und Blutuntersuchungen stattgefunden sowie mehrere Sonografien, zudem eine Magen- und Darmspiegelung und zuletzt bei geringfügig vergrößerten mesenterialen Lymphknoten auch eine Laparoskopie mit prophylaktischer Entfernung der Appendix. Die Ärzte hätten der Familie dennoch immer wieder gesagt, dass Greta gesund sei, und sie nach Hause geschickt. Stress als Auslöser habe die Familie 
ausschließen können, da die Symptomatik sich auch in stressfreien Zeiten, wie den Ferien, gezeigt habe.

Die Eltern seien weiterhin der Überzeugung, eine schwere Erkrankung könne übersehen werden. Über eigene belastende Erfahrungen zu sprechen, lehnen beide Eltern mit dem Hinweis „nicht vor dem Kind“ ab. In späteren Gesprächen berichten die Eltern, dass es vor Gretas Geburt eine Fehlgeburt im 6. Schwangerschaftsmonat gegeben habe, sowie ein Geschwisterkind, das mit drei Monaten am plötzlichen Kindstod verstorben sei. Greta habe deshalb das erste Lebensjahr am Monitor verbracht, die Mutter habe in dieser Zeit Gretas Wohlbefinden häufig mit dem Monitor in Verbindung gebracht, sie habe ihrer Intuition, was das Kind brauche, nicht mehr trauen können. Greta habe sich gut entwickelt, sie sei ehrgeizig und komme gut zurecht, sie sei allerdings immer eher ängstlich gewesen.

Vor Beginn von Gretas Symptomatik sei die Mutter erneut schwanger gewesen, sie habe das Kind jedoch vor Ende des dritten Schwangerschaftsmonats verloren. Greta habe davon nichts mitbekommen. Die Mutter habe ein paar Tage in der Klinik verbringen müssen, der Vater habe Greta in dieser Zeit betreut. Greta habe man gesagt, die Mutter sei wegen Bauchschmerzen in der Klinik.

Aufgrund der chronifizierten Symptomatik wird Greta vollstationär kinderpsychiatrisch aufgenommen. In einer Vielzahl therapeutischer Gespräche, gelingt es Greta und ihren Eltern langsam, sich auf eine psychosomatische Krankheitsgenese einzulassen.

Vereinbartes Ziel ist es zunächst, den Alltag trotz der Bauchschmerzen wieder mitmachen zu können. Hier zeigt sich schnell eine Diskrepanz zwischen angegebenen Schmerzen und der dennoch bestehenden Alltagstauglichkeit im Stationsalltag. Zusätzlich wird an der Bewältigung des Schmerzes gearbeitet. Wichtige Eckpunkte sind zweimal wöchentliche, vom Schmerz unabhängige Termine bei der Stationsärztin sowie verordnete Ruhezeiten in der Hängematte mit Wärmflasche und Hörspiel. Greta kann selbst gut formulieren, welche Therapie sie als hilfreich empfinden würde, so gelingt ihr zum Einen eine Verringerung der Beschwerden, ohne dass sie ihr Gesicht verliert, zum Anderen erlebt sie sich selbst als wirksam beteiligt an der Veränderung.

\section{Tabelle 4}

Dos and Don'ts.

\begin{tabular}{|ll|}
\hline Dos & Don'ts \\
\hline Vorbefunde zusammenfassen & Doppeluntersuchungen \\
\hline $\begin{array}{l}\text { Untersuchungen auf das Nötigste } \\
\text { begrenzen, mit zeitlicher Straffung, } \\
\text { Endpunkte von vornherein festlegen }\end{array}$ & $\begin{array}{l}\text { weitere Untersuchungen vorschlagen, } \\
\text { Überweisungen zu weiteren Spezialisten }\end{array}$ \\
\hline $\begin{array}{l}\text { transparente, entkatastrophisierende } \\
\text { Besprechungen, Normvarianten/ } \\
\text { Bagatellbefunde einordnen }\end{array}$ & Bagatellbefunde uneingeordnet mitteilen \\
\hline
\end{tabular}

schnelle Reintegration in den Alltag

fortgesetzte Krankschreibungen

interdisziplinäre Zusammenarbeit mit Anerkennung der Beschwerden bzw. frühzeitiger Einschaltung eines Kinderund Jugendpsychiatrischen Konsiliardienstes oder einer Zusammenarbeit mit niedergelassenen Kinder- und Jugendpsychiatern

einen gesichtswahrenden „Rückzug in Würde“ über einen längeren Zeitraum ermöglichen

Aussagen treffen wie: „Wir können nichts für sie tun“, „Sie simulieren“. Chronifizierung durch Weiterbehandlung trotz somatisch unauffälligem Befund
Reflektion über eigene Gefühle im Zusammenhang mit der Erwartung des Patienten (Idealisierung/Entwertung/ „Koryphäenkiller“)
Verlegenheitsverordnung von Schmerzmedikation, längerfristige Physiotherapie, Placebobehandlung (vor allem zur „Überführung“ von Patienten bei Simulationsvermutung)

Ohnmacht/Wut direkt und ungefiltert dem Patienten gegenüber in Kontakt bringen

\section{Infobox Medikation (nach [15])}

- Selektive Serotoninwiederaufnahmehemmer (SSRI) und selektive Serotonin- und Noradrenalinwiederaufnahmehemmer (SSNRI) scheinen eine positive Wirkung auf die Zielsymptomatik, vorrangig die Schmerzsymptomatik zu haben, es gibt jedoch keine placebokontrollierten Studien zur Wirksamkeit.

- Im Kindesalter gibt es keine Zulassung bei somatoformen Störungen, sodass individuelle Heilversuche im Off-LabelBereich notwendig werden; lediglich bei einer komorbiden depressiven Episode ist Fluoxetin ab 8 Jahren zugelassen.

- Im Erwachsenenalter ist Opipramol (ein trizyklisches Piperazinylderivat) bei somatoformen Störungen zugelassen, es zeigt vorrangig auf Anspannung und begleitende Ängste Wirkung.

- Insgesamt ist bei einer erhöhten somatischen Wahrnehmung und Empfindlichkeit von Patienten mit somatoformen Störungen von einer höheren Rate an unerwünschten Nebenwirkungen auszugehen. 
Eine Übertragung der Symptomatik in eine Metapher „was schlägt dir auf den Magen“ kann nur im geschützten therapeutischen Rahmen versucht werden. Greta ist hier sehr deutlich und benennt massive Ängste um die Mutter und Sorgen vor dem eigenen Tod.

Um eine Übertragung in den Alltag außerhalb der Klinik zu gewährleisten, wird mit den Eltern zum einen in Video-Interaktionen an der nonverbalen Signalwahrnehmung von Greta gearbeitet, zum anderen das richtige Reagieren besprochen, wenn der Schmerz kommt (echtes Mitgefühl und Trost, ohne Schonung und Vergünstigungen). Auch die Kommunikation wichtiger Familienthemen wird geübt. Die Mutter begibt sich in eine eigene Psychotherapie, Greta beginnt ebenfalls nachstationär eine Psychotherapie; kinderpsychiatrische Verlaufskontrollen finden weiterhin regelmäßig statt.

\section{Fallbeispiel 2: „Wenn ich jetzt in die Psychiatrie gehe, denken alle, ich hab was am Kopf“"}

Der 17-jähirge Nico, der gerade eine Ausbildung zum KFZ-Mechaniker machte, wurde auf dem Weg zur Arbeit ohne erkennbare Ursache ohnmächtig. Nach dem Erwachen konnte er seine Beine nicht bewegen und spürte sie auch nicht. Es folgte eine ausgiebige kinderärztliche Diagnostik und Behandlung, einschließlich intensiver neurologischer Diagnostik, die allesamt keine Befunde erbrachten, welche das Problem angemessen erklären würden. Dennoch blieben die Lähmung und die Sensibilitätseinbuße, sodass Nico sogar für einige Wochen in die neurologische Reha ging. Die Lähmung war im Verlauf rückläufig und er konnte auf den Rollstuhl verzichten, während die Sensibilitätseinbußen bei regelhafter NLG und Fehlen jeglicher somatischer Erklärung fortdauerten. Aufgrund des hohen Leidensdrucks erfolgte die stationäre Aufnahme auf die Kinder- und Jugendpsychiatrische Station bei V.a. eine dissoziative Störung. Der während des Aufenthalts volljährig gewordene Patient zeigte zum Teil eine kindliche Bedürfnislage nach Anlehnung und Versorgung bei einem gleichzeitigen Idealbild einer ausgeprägten Selbstständigkeit. Ähnlich zeigte sich Nico im familiären Umfeld, wo er im Zusammenhang mit der alleinerziehenden Mutter eine ähnliche Bedürftigkeit ausdrückte.
Aufgrund seiner ausgeprägten ehrenamtlichen Tätigkeiten und Erfahrungen bei einem sozialen Träger, zeigte sich Nico häufig fachmännisch, zuverlässig, betont erwachsen und reif, was wenig mit dem korrespondierte, wie man ihn wahrnahm. Anfangs lehnte er gänzlich psychische Ursachen der derzeitigen Beeinträchtigungen $\mathrm{ab}$ und vermittelte eine technische Überzeugung, dass sein Problem sicher bald weg ginge. Es mache ihn „fertig“, dass er deswegen in eine Psychiatrie müsse; seine Bekannten müssten dann evtl. denken, dass er etwas „am Kopf“ habe.

Im Rahmen des stationären Aufenthalts lernte Nico zunehmend, über für ihn wenig zugängliche Empfindungen zu reflektieren, eigene Überforderungen wahrzunehmen und zu tolerieren, Bedürfnisse angemessen zu äußern und in einen zwischenmenschlichen Bezug zu treten, der freier war von den vorigen Haltungen der scheinbaren Überlegenheit. Tatsächlich sprach er immer weniger von seinen Sensibilitätseinbußen, sodass er am Ende seiner Behandlung überrascht darüber war, wahrzunehmen, dass die Gefühlsstörungen nicht mehr vorhanden waren.

\section{Fazit}

Die hochkomplexe Entwicklung von Fähigkeiten, Wissen und psychischer Struktur im Kindes- und Jugendalter vollzieht sich in prägenden Beziehungen zu relevanten Bindungs- und Bezugspersonen im umgebenden soziokulturellen Kontext und geht mit der erfahrungsabhängigen neuroplastischen Reifung des Gehirns einher. Psychische und psychosomatische Störungen stellen daher immer auch ein Risiko für die weitere Persönlichkeits- und Bildungsentwicklung dar. Ihre Untersuchung und Behandlung sollte deshalb primär durch Kinder- und Jugendpsychiater erfolgen. Der regelhaften kinderärztlichen Versorgung kommen dabei wesentliche und oft weichenstellende Aufgaben zu. 


\section{Über die Autoren}

\section{Stephan Anis Towfigh}

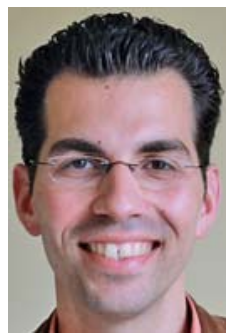

Jahrgang 1981, Dr. med.; Facharzt für Kinder- und Jugendpsychiatrie und Psychotherapie. 2006 Promotion in der Geschichte der Medizin. Seit 2013 leitender Oberarzt der Klinik für Kinder- und Jugendpsychiatrie, Psychotherapie und Psychosomatik am Ernst von Bergmann Klinikum in Potsdam. Regelmäßige konsiliarische Tätigkeit in den pädiatrischen Abteilungen des Hauses, insbesondere auch ambulante Versorgung von psychosomatisch erkrankten Kindern und Jugendlichen. Weitere Schwerpunkte: Psychodynamische und strukturbezogene Psychotherapie, Psychoneuroimmunologie, Therapeutische Betreuung von Kindern und Jugendlichen mit psychiatrischen Auffälligkeiten im Kontext von Fluchterfahrungen.

\section{Lena Kuntze}

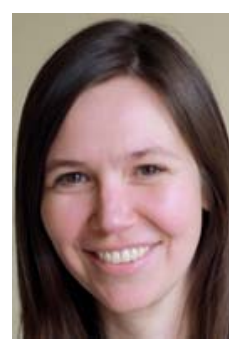

Jahrgang 1975, Dr. med.; Fachärztin für Kinder- und Jugendpsychiatrie und Psychotherapie. 2006 Promotion. Facharztausbildung an den Kliniken für Kinder- und Jugendpsychiatrie, Psychotherapie und Psychosomatik am Evangelischen Krankenhaus Herzberge und an der Charité Berlin. Seit 2014 Oberärztin der Kinderstation und der gruppentherapeutischen Station in der Klinik für Kinder- und Jugendpsychiatrie, Psychotherapie und Psychosomatik am Ernst von Bergmann Klinikum, Potsdam. Regelmäßige konsiliarische Tätigkeit in den pädiatrischen Abteilungen des Hauses, insbesondere auch ambulante Versorgung von psychosomatisch erkrankten Kindern und Jugendlichen; weitere Schwerpunkte: Psychodynamische und strukturbezogene Psychotherapie, Eltern-Kind-Interaktion, Traumatherapie, Kinderschutz.

\section{Michael von Aster}

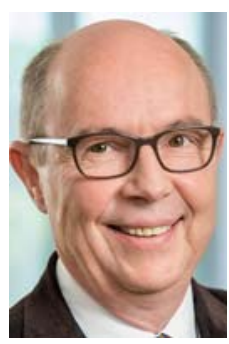

Jahrgang 1951, Prof. Dr. med., Dipl.Päd.; Facharzt für Kinder- und Jugendpsychiatrie, Psychotherapie und Psychosomatik, Chefarzt der Kliniken für Kinder- und Jugendpsychiatrie, Psychotherapie und Psychosomatik an den DRK Kliniken Berlin I Westend und am Ernst von Berg- mann Klinikum Potsdam, Honorarprofessor Universität Potsdam. Studium der Erziehungswissenschaften und der Humanmedizin an der Freien Universität Berlin, 1985 Promotion, 1998 Habilitation Universität Zürich. Bis 2003 Leitender Arzt am Zentrum für Kinder- und Jugendpsychiatrie Universität Zürich. Wissenschaftliche Schwerpunkte: Ursachen, Verlauf, Diagnostik und Therapie Schulischer Entwicklungsstörungen, Forschungsgruppen am Zentrum für Neurowissenschaften und Kinderspital der Universität Zürich und am Institut für Klinische Psychologie der Universität Potsdam.

\section{Korrespondenzadresse}

Prof. Dr. Michael von Aster

Klinik für Kinder- und Jugendpsychiatrie, Psychosomatik und Psychotherapie

DRK Kliniken Berlin | Westend

Spandauer Damm 130

14050 Berlin

E-Mail: m.aster@drk-kliniken-berlin.de

Interessenkonflikt: Die Autoren geben an, dass kein Interessenkonflikt besteht.

\section{Literatur}

1 Essau CA, Conradt J, Petermann F. Häufigkeit und Komorbidität somatoformer Störungen bei Jugendlichen: Ergebnisse der Bremer Jugendstudie. Z Klin Psychol Psychotherapie 2000; 29: $97-108$

2 Huertas-Ceballos AA, Logan S, Bennett C et al. Dietary interventions for recurrent abdominal pain (RAP) and irritable bowel syndrome (IBS) in childhood. Cochrane Database Syst Rev 2009; 1: CD003019

3 Tomasson K, Kent D, Coryell W. Somatization and conversion disorders: comorbidity and demographics at presentation. Acta Psychiatr Scand 1991; 84: $218-228$

4 Winter S, Köberle C, Lenz K et al. Systematik somatoformer Störungen. Monatsschr Kinderheilkd 2012; 160 : 20-26

5 Rudolf G. Strukturbezogene Psychotherapie: Leitfaden zur psychodynamischen Therapie struktureller Störungen. Stuttgart: Schattauer; 2009: 13-17

6 Grossmann KE. Skalen zur Erfassung mütterlichen Verhaltens von Mary D.S. Ainsworth. In: Grossmann KE (Hrsg). Entwicklung der Lernfähigkeit in der sozialen Umwelt. München: Kindler; 1977: 96-107

7 Noeker M. Funktionelle und somatoforme Störungen des Kindes- und Jugendalter. Göttingen: Hogrefe; 2008: $191 \mathrm{ff}$

8 Schubert C (Hrsg). Psychoneuroimmunologie und Psychotherapie. Stuttgart: Schattauer; 2015: $82 \mathrm{ff}$

9 Schubert C (Hrsg). Psychoneuroimmunologie und Psychotherapie. Stuttgart: Schattauer; 2015: 7

10 Noeker M. Funktionelle und somatoforme Störungen des Kindes- und Jugendalter. Göttingen: Hogrefe; 2008: $30 \mathrm{ff}$ 
11 Deter C. Angewandte Psychosomatik: Eine Anleitung zum Erkennen, Verstehen und behandeln psychosomatisch Kranker. Stuttgart: Thieme; 1997

12 Schultz-Venrath U. Lehrbuch Mentalisieren: Psychotherapien wirksam gestalten. Stuttgart: Klett-Cotta; 2013

13 Emich-Widera E, Kazek B, Szwed-Białożyt B et al. Headaches as Somatoform Disorders in Children and Adolescents. Ment Illn 2012; 4: e9

14 Noeker M. Funktionelle und somatoforme Störungen des Kindes- und Jugendalter. Göttingen: Hogrefe; 2008

15 Benkert O, Hippius $\mathrm{H}$. Kompendium der psychiatrischen Psychopharmakotherapie. 10. Aufl. Heidelberg: Springer; 2014: $28 \mathrm{ff}$

\section{Weiterführende Literatur}

Beesdo K. Pain associated with specific anxiety and depressive disorders in a nationally representative population sample. Soc Psychiatry Psychiatr Epidemiol 2010; 45 : 89-104

Brunner R, Resch F. Dissoziative und somatoforme Störungen. In: Herpertz-Dahlmann B, Resch F, Schulte-Markwort M, Warnke A (Hrsg). Entwicklungspsychiatrie. Stuttgart: Schattauer; 2008: $940-969$
Charlier N. Somatische Differentialdiagnosen psychischer Symptome im Kindes- und Jugendalter. Heidelberg: Springer; 2008

Hallett M. Functional (psychogenic) movement disorders Clinical presentations. Parkinsonism Relat Disord 2016; 22 $149-152$

Herpertz-Dahlmann B, Warnke A (Hrsg). Psychosomatisches Kompendium der Pädiatrie. Leitfaden für den Kinder- und Jugendarzt. München: Hans Marseille; 2006

Patel S, Shaw RJ, Frank R. Somatoforme Störungen. In: Lehmkuhl G, Poustka F, Holtmann M, Steiner H (Hrsg). Lehrbuch der Kinder- und Jugendpsychiatrie. Göttingen: Hogrefe; 2013: 917 - 943

Van der Hoeven RM, Broersma M, Pijnenborg GH et al. Functional (psychogenic) movement disorders associated with normal scores in psychological questionnaires: A case control study. J Psychosom Res 2015; 79 : 190 - 194

Tegethoff M, Belardi A, Stalujanis E et al. Comorbidity of Mental Disorders and Chronic Pain: Chronology of Onset in Adolescents of a National Representative Cohort. J Pain 2015; $16: 1054-1064$ 


\section{CME-Fragen}

\section{CME.thieme.de}

CME-Teilnahme

- Viel Erfolg bei Ihrer CME-Teilnahme unter http://cme.thieme.de

- Diese Fortbildungseinheit ist 12 Monate online für eine CME-Teilnahme verfügbar.

- Sollten Sie Fragen zur Online-Teilnahme haben, unter

http://cme.thieme.de/hilfe finden Sie eine ausführliche Anleitung.

1

Das Phänomen der

„belle indifférrence“bedeutet...
A das Simulieren des Patienten.
B das Missverhältnis zwischen dem Arzt und dem Patienten.
C das Missverhältnis zwischen den objektivierbaren somatischen Befunden und dem erlebten Leid des Patienten.
D die Präsentation des Schmerzes mit einem unpassenden, häufig wenig leidenden Affekt.
E die Gleichgültigkeit des Patienten gegenüber positiven Erlebnissen.

\section{2}

A Der Beginn einer somatoformen Problematik steht häufig in zeitlichem Zusammenhang mit für den Patienten belastenden Ereignissen.

B Eltern ist häufig der zeitliche Zusammenhang zwischen psychischen Belastungen und dem Auftreten einer Störung nicht bewusst.

C Häufig gibt es auch in der Familie des Jugendlichen ähnliche somatoforme Störungsmuster.

D Es sollte sofort und zu einem frühen Zeitpunkt konfrontiert werden, dass es sich bei den Störungen um eine psychosomatische Erkrankung handelt.

E Häufig liegen aktuelle psychiatrische oder psychosoziale Belastungsfaktoren bei den Eltern vor.

\section{3}

Bezüglich des historischen Verständnisses über somatoforme Störungen trifft Folgendes zu:
A C.G. Jung war der erste, der das Konzept der Psychosomatik formulierte.

B Sigmund Freud prägte den Begriff „Psychosomatik“.

C Franz Alexander beschrieb psychosomatische Patienten als sanguinische Persönlichkeiten.

D Alfred Adler war der erste, der körperliche von somatoformen Erkrankungen unterschied.

E Sigmund Freud formulierte die Idee, dass unerträgliche Vorstellungen körperlich ausgedrückt werden könnten.
Bei katastrophisierenden Schmerzwahrnehmungen sind folgende Phänomene nicht vorzufinden:
A Die Symptomatik wird übersteigert wahrgenommen und geäußert.

B Die Symptomatik wird auch auf andere Bereiche generalisiert.

C Es entsteht ein Gefühl der Hilflosigkeit und des Ausgeliefertseins.

D Es ist kaum möglich, sich auf andere Dinge zu konzentrieren, da die gesamte Aufmerksamkeit auf die Beschwerden gelenkt wird.

E Die Symptomatik wird vom Patienten bagatellisiert.

\section{5}

Andauernder, schwerer, quälender Schmerz, der nicht oder nicht hinreichend durch eine physiologische Störung erklärt werden kann, wird in der ICD-10 definiert als...
A anhaltende Schmerzstörung
B Konversionsstörung
C Dissoziative Fugue
D Simulation
E Münchhausen by Proxy 


\section{CME-Fragen}

Psychosomatische Störungen im Kindes- und Jugendalter

\section{6}

Was ist bezüglich der elterlichen Antwort in der Eltern-Kind-Interaktion zutreffend?
A Sie ist für das Kind unerheblich.
B Sie entscheidet mit darüber, welchen Affekt das Kind erlebt.
C Ihre Voraussagbarkeit ist für die Bewusstseinsentwicklung des Kindes nicht von Bedeutung.
D Sie ist durch Erkrankungen der Eltern nie beeinträchtigt.
E Sie sollte nur aus einer verbalen Antwort bestehen.

7

Welche Aussage zur dissoziativen Störung trifft nicht zu?
A Sie steht häufig mit einer Traumatisierung in Zusammenhang.
B Sie kann mit einer ähnlichen Dramatik wie ein epileptischer Anfall imponieren.
C Die dissoziativen Sensibilitätsstörungen folgen häufig nicht den neurologischen Grenzen.
D Sie kann sich als Sehstörung, Gedächtnisverlust oder Gangstörung äußern.
E Die dargebotenen Symptome sind nicht variabel.

8

Welche Aussage zur HPA-Achse trifft nichtzu?
A Ihre Aktivierung beeinflusst die Nebennierenrinde.
B Sie beeinflusst die Rückregulierung Stress-getriggerter proinflammatorischer Zytokine.
C Bei dauerhafter Aktivierung kommt es zunächst zu einem Hypokortisolismus und in der Folge zu einem Hyperkortisolismus.
D Sie kann aufgrund psychischen Stresses chronisch aktiviert sein.
E Bei Abnahme ihrer Funktionstüchtigkeit kann es in der Folge zu einer „silent inflammation“ kommen.

Wann sollte unabdingbar eine Übergabe an einen Kinder- und Jugendpsychiater erfolgen?
A Bei guter schulischer und sozialer Integration.
B Bei gestörter Wahrnehmung der Eltern, im Sinne einer Somatoformen Störung by Proxy.
C Bei kurzer Krankheitsdauer und schneller Remission.
D Bei nicht abgeschlossener somatischer Untersuchung.
E Bei monosymptomatischem Krankheitsbild.

10

Was ist förderlich für die Arzt-Patienten-Beziehung bei Somatoformen Störungen?
A Zeitdruck
B wechselnder Ansprechpartner
C Bagatellisierung der Symptomatik
D Einbeziehung der Patienten in die Therapie
E Meidung von körperlichen Untersuchungen 